\title{
Left-symmetric Bialgebras and An Analogue of the Classical Yang-Baxter Equation
}

\author{
Chengming Bai ${ }^{1,2}$ \\ 1. Chern Institute of Mathematics $\&$ LPMC, Nankai University, Tianjin 300071, P.R. China \\ 2. Department of Mathematics, Rutgers, The State University of New Jersey, Piscataway, NJ \\ 08854, U.S.A.
}

\begin{abstract}
We introduce a notion of left-symmetric bialgebra which is an analogue of the notion of Lie bialgebra. We prove that a left-symmetric bialgebra is equivalent to a symplectic Lie algebra with a decomposition into a direct sum of the underlying vector spaces of two Lagrangian subalgebras. The latter is called a parakähler Lie algebra or a phase space of a Lie algebra in mathematical physics. We introduce and study coboundary left-symmetric bialgebras and our study leads to what we call "S-equation", which is an analogue of the classical Yang-Baxter equation. In a certain sense, the $S$-equation associated to a left-symmetric algebra reveals the left-symmetry of the products. We show that a symmetric solution of the $S$-equation gives a parakähler Lie algebra. We also show that such a solution corresponds to the symmetric part of a certain operator called "O-operator", whereas a skew-symmetric solution of the classical YangBaxter equation corresponds to the skew-symmetric part of an $\mathcal{O}$-operator. Thus a method to construct symmetric solutions of the $S$-equation (hence parakähler Lie algebras) from $\mathcal{O}$ operators is provided. Moreover, by comparing left-symmetric bialgebras and Lie bialgebras, we observe that there is a clear analogue between them and, in particular, parakähler Lie groups correspond to Poisson-Lie groups in this sense.
\end{abstract}

Key Words parakähler Lie algebra, left-symmetric algebra, left-symmetric bialgebra, $S$ equation

Mathematics Subject Classification 17B, 53C, 81R 


\section{Introduction}

Left-symmetric algebras (or under other names like pre-Lie algebras, quasi-associative algebras, Vinberg algebras and so on) are Lie-admissible algebras ((nonassociative) algebras whose commutators are Lie algebras) whose left multiplication operators form a Lie algebra. They have already been introduced by A. Cayley in 1896 as a kind of rooted tree algebras ([Ca]). They also arose from the study of several topics in geometry and algebra in 1960s, such as convex homogenous cones ([V]), affine manifolds and affine structures on Lie groups ([Ko],[Mat]), deformation of associative algebras ([G]) and so on. In particular, a Lie algebra $\mathcal{G}$ with a compatible left-symmetric algebra structure is the Lie algebra of a Lie group $G$ with a left-invariant affine structure, that is, there exists a left-invariant (locally) flat and torsion free connection $\nabla$ in $G$. The left-symmetric algebra structure corresponds to the connection $\nabla$ given by $X Y=\nabla_{X} Y$ for $X, Y \in \mathcal{G}$ and (a geometric interpretation of) the left-symmetry is just the flatness of the connection $\nabla([\mathrm{Me}],[\mathrm{Ki}])$.

Furthermore, as it was pointed out in [CL] by Chapoton and Livernet, the left-symmetric algebra "deserves more attention than it has been given". It appears in many fields in mathematics and mathematical physics. In [Bu2], Burde gave a survey of certain different fields in which left-symmetric algebras play an important role, such as vector fields, rooted tree algebras, words in two letters, vertex algebras, operad theory, deformation complexes of algebras, convex homogeneous cones, affine manifolds, left-invariant affine structures on Lie groups (see [Bu2] and the references therein). Here are some more examples (partly overlap with some examples in $[\mathrm{Bu} 2])$.

(a) Symplectic structures on Lie groups and Lie algebras. A symplectic Lie group is a Lie group $G$ with a left-invariant symplectic form $\omega^{+}$. One can define an affine structure on $G$ by $([\mathrm{Ch}])$

$$
\omega^{+}\left(\nabla_{x^{+}} y^{+}, z^{+}\right)=-\omega^{+}\left(y^{+},\left[x^{+}, z^{+}\right]\right)
$$

for any left-invariant vector fields $x^{+}, y^{+}, z^{+}$and hence $x^{+} y^{+}=\nabla_{x^{+}} y^{+}$gives a left-symmetric algebra. In fact, equation (1.1) is of great importance to the study of symplectic and Kähler Lie groups $([\mathrm{H}],[\mathrm{LM}],[\mathrm{DaM} 1-2],[\mathrm{MS}],[\mathrm{KGM}])$.

(b) Complex and complex product structures on Lie groups and Lie algebras. From a real left-symmetric algebra $A$, it is natural to define a Lie algebra structure on the vector space $A \oplus A$ 
(that is $\left.\mathcal{G}(A) \ltimes_{L} \mathcal{G}(A)\right)$ such that

$$
J(x, y)=(-y, x), \quad \forall x, y \in A
$$

is a complex structure on it. Moreover, there is a correspondence between left-symmetric algebras and complex product structures on Lie algebras ([AS]), which plays an important role in the theory of hypercomplex and hypersympletic manifolds ([Bar],[AD]).

(c) Vertex algebras. Vertex algebras are fundamental algebraic structures in conformal field theory $([\mathrm{FLM}],[\mathrm{FHL}],[\mathrm{JL}])$. For any vertex algebra $V$,

$$
a * b=a_{-1} b, \quad \forall a, b \in V
$$

defines a left-symmetric algebra. And a vertex algebra is equivalent to a left-symmetric algebra and a Lie conformal algebra with some compatibility conditions ([BK]).

Vertex algebras are also closely related to a subclass of (finite-dimensional) left-symmetric algebras, namely, Novikov algebras. Novikov algebras are left-symmetric algebras with commutative right multiplication operators. They were introduced in connection with Hamiltonian operators in the formal variational calculus ([GD]) and the following Poisson brackets of hydrodynamic type $([\mathrm{BN}])$

$$
\left\{u\left(x_{1}\right), v\left(x_{2}\right)\right\}=\frac{\partial}{\partial x_{1}}\left((u v)\left(x_{1}\right)\right) x_{1}^{-1} \delta\left(\frac{x_{1}}{x_{2}}\right)+(u v+v u)\left(x_{1}\right) \frac{\partial}{\partial x_{1}} x_{1}^{-1} \delta\left(\frac{x_{1}}{x_{2}}\right) .
$$

Furthermore, let $A$ be a Novikov algebra and set $\mathcal{A}=A \otimes \mathbf{C}\left[t, t^{-1}\right]$, where $t$ is an indeterminate. Then the bracket

$$
\left[a \otimes t^{m}, b \otimes t^{n}\right]=(-m a b+n b a) \otimes t^{m+n-1}, \quad \forall a, b \in A, m, n \in \mathbf{Z}
$$

defines a Lie algebra structure on $\mathcal{A}$ and this Lie algebra can be used to construct a vertex Lie algebra and a vertex algebra $([\mathrm{Li}])$. Conversely, vertex algebras satisfying certain conditions must correspond to some Novikov algebras (roughly speaking, such a vertex algebra $V$ is generated from $V_{(2)}$ which is a Novikov algebra, with some additional conditions) ([BKL]).

(d) Phase spaces of Lie algebras. The concept of phase space of a Lie algebra was introduced by Kupershmidt in [Ku1] by replacing the underlying vector space with a Lie algebra and was generalized in [Bai2]. In [Ku2], Kupershmidt pointed out that left-symmetric algebras appear as an underlying structure of those Lie algebras that possess a phase space and thus they form a natural category from the point of view of classical and quantum mechanics. 
(e) Left-symmetric algebras are closely related to certain integrable systems ([Bo1], [SS],[W]), classical and quantum Yang-Baxter equation ([ES],[Ku3],[GS],[DiM]), combinatorics ([E]) and so on. In particular, they play a crucial role in the Hopf algebraic approach of Connes and Kreimer to renormalization of perturbative quantum field theory $([\mathrm{CK}])$.

In this paper, we study a structure, namely, parakähler structure, which appears in both geometry and mathematical physics, in terms of left-symmetric algebras. In geometry, a parakähler manifold is a symplectic manifold with a pair of transversal Lagrangian foliations ([Lib]). A parakähler Lie algebra $\mathcal{G}$ is the Lie algebra of a Lie group $G$ with a $G$-invariant parakähler structure $([\mathrm{Ka}])$. It is a symplectic Lie algebra with a decomposition into a direct sum of the underlying vector spaces of two Lagrangian subalgebras. Some basic facts on the parakähler structures on Lie groups and Lie algebras have been given in [Bai4]. On the other hand, a phase space of a Lie algebra in mathematical physics ([Ku1-2], [Bai2]) is a parakähler Lie algebra. We will show in this paper that conversely every parakähler Lie algebra is isomorphic to a phase space of a Lie algebra.

We have obtained a structure theory of parakähler Lie algebras in terms of matched pairs of Lie algebras (cf. Theorem 2.5) in [Bai2] and [Bai4]. This theory in fact gives a construction of parakähler Lie algebras. However, except for some examples, it is still unclear when the compatibility conditions appearing in the structure theory are satisfied.

The aim of this paper is to study further the structures of parakähler Lie algebras or phase spaces of Lie algebras in terms of left-symmetric algebras and interpret the construction mentioned above using certain equivalent conditions which are much easier to use. Briefly speaking, a parakähler Lie algebra is equivalent to a certain bialgebra structure, namely, a left-symmetric bialgebra structure. From the point of view of phase spaces of Lie algebras, such a structure seems to be very similar to the Lie bialgebra structure given by Drinfeld ([D]). In fact, left-symmetric bialgebras have many properties similar to those of Lie bialgebras. In particular, there are so-called coboundary left-symmetric bialgebras which lead to an analogue ( $S$-equation) of the classical Yang-Baxter equation. In a certain sense, the $S$-equation in a left-symmetric algebra reveals the left-symmetry of the products. A symmetric solution of the $S$-equation gives a parakähler Lie algebra.

Furthermore, comparing left-symmetric bialgebras and Lie bialgebras in terms of several different properties, we observe that there is a clear analogy between them and in particular, parakähler Lie groups correspond to Poisson-Lie groups whose Lie algebras are Lie bialgebras in 
this sense. Since the classical Yang-Baxter equation can be regarded as a "classical limit" of the quantum Yang-Baxter equation ([Be]), the analogy mentioned above, especially, the $S$-equation corresponding to the classical Yang-Baxter equation found in this paper, suggests that there might exist an analogue ("quantum $S$-equation") of the quantum Yang-Baxter equation. The results in this paper are the beginning of a program to develop the theory of such analogues of the quantum Yang-Baxter equation. We expect that our future study will be related to the theory of quantum groups, tensor categories and vertex operator algebras.

We would like to point out that many structures (for example, see Theorem 3.8, Theorem 5.4 and so on) appearing in this paper exhibit features of both Lie algebras and left-symmetric algebras, although the study of parakähler Lie algebras seems to be purely a topic in Lie algebras. Indeed, the theory of Lie algebras alone is not enough here. Hence, unlike the theory of Lie bialgebras which is purely Lie-algebra-theoretic, we need to combine the ideas and methods from both the theory of Lie algebras and the theory of left-symmetric algebras.

The paper is organized as follows. In Section 2, we give some necessary definitions and notations and basic results on left-symmetric algebras and parakähler Lie algebras. In Section 3 , we study how to construct a left-symmetric algebra which is the direct sum of two leftsymmetric subalgebras. We observe that in the case of parakähler Lie algebras, matched pairs of left-symmetric algebras are equivalent to the corresponding matched pairs of their sub-adjacent Lie algebras, whereas it is not true in general. This also partly explains why left-symmetric algebras appear in a problem which seems to be purely Lie-algebra-theoretic. In Section 4, we introduce the notion of left-symmetric bialgebra which is precisely equivalent to the notion of parakähler Lie algebra. In Section 5, we consider the special case that a certain 1-cocycle appearing in a left-symmetric bialgebra is coboundary. A sufficient and necessary condition for the existence of such a structure leads to certain explicit equations. In Section 6, we discuss only the simplest cases in Section 5. We obtain an equation in the left-symmetric algebra, namely, the $S$-equation, which is an analogue of the classical Yang-Baxter equation in a Lie algebra. We also give some important properties of the $S$-equation. In Section 7, we compare left-symmetric bialgebras and Lie bialgebras by recalling some facts on Lie bialgebras. We also consider the case that a left-symmetric bialgebra is also a Lie bialgebra.

Throughout this paper, all algebras are finite-dimensional, although many results still hold in the infinite-dimensional case. 


\section{Preliminaries and basic results}

Definition 2.1 Let $A$ be a vector space over a field $\mathbf{F}$ with a bilinear product $(x, y) \rightarrow x y$. $A$ is called a left-symmetric algebra if for any $x, y, z \in A$, the associator

$$
(x, y, z)=(x y) z-x(y z)
$$

is symmetric in $x, y$, that is,

$$
(x, y, z)=(y, x, z), \text { or equivalently }(x y) z-x(y z)=(y x) z-y(x z)
$$

Left-symmetric algebras are Lie-admissible algebras (cf. [Me]).

Proposition 2.1 Let $A$ be a left-symmetric algebra. For any $x, y \in A$, let $L_{x}$ and $R_{x}$ denote the left and right multiplication operator respectively, that is, $L_{x}(y)=x y, R_{x}(y)=y x$. Let $L: A \rightarrow g l(A)$ with $x \rightarrow L_{x}$ and $R: A \rightarrow g l(A)$ with $x \rightarrow R_{x}$ (for every $x \in A$ ) be two linear maps. Then we have the following results:

(1) The commutator

$$
[x, y]=x y-y x, \quad \forall x, y \in A,
$$

defines a Lie algebra $\mathcal{G}(A)$, which is called the sub-adjacent Lie algebra of $A$ and $A$ is also called the compatible left-symmetric algebra structure on the Lie algebra $\mathcal{G}(A)$.

(2) $L$ gives a regular representation of the Lie algebra $\mathcal{G}(A)$, that is,

$$
\left[L_{x}, L_{y}\right]=L_{[x, y]}, \quad \forall x, y \in A
$$

(3) The identity (2.2) is equivalent to the following equation

$$
\left[L_{x}, R_{y}\right]=R_{x y}-R_{y} R_{x}, \quad \forall x, y \in A
$$

Left-symmetric algebras can be obtained from some known algebraic structures (it can be regarded as a "realization theory"). Recall that a Novikov algebra $A$ is a left-symmetric algebra satisfying $R_{x} R_{y}=R_{y} R_{x}$ for any $x, y \in A$.

Example 2.1 Let $(A, \cdot)$ be a commutative associative algebra and $D$ be its derivation. Then the new product

$$
x *_{a} y=x \cdot D y+a \cdot x \cdot y, \quad \forall x, y \in A
$$

makes $\left(A, *_{a}\right)$ become a Novikov algebra for $a=0$ by S. Gel'fand ([GD]), for $a \in \mathbf{F}$ by Filipov ([F]) and for a fixed element $a \in A$ by $\mathrm{Xu}([\mathrm{X}])$. In [BM2-3], we constructed a deformation 
theory of Novikov algebras. In particular, the two kinds of Novikov algebras given by Filipov and $\mathrm{Xu}$ are the special deformations of the algebras $(A, *)=\left(A, *_{0}\right)$ given by $\mathrm{S}$. Gel'fand. Moreover, we proved that the Novikov algebras in dimension $\leq 3$ ([BM1]) can be realized as the algebras defined by S. Gel'fand and their compatible linear deformations. We conjectured that this conclusion can be extended to higher dimensions. On the other hand, due to the structures of free Novikov algebras, any Novikov algebra is a quotient of a subalgebra of an (infinite-dimensional) algebra given by equation (2.6) for $a=0$ ([DL]).

Example 2.2 Let $(\mathcal{G},[]$,$) be a Lie algebra and R: \mathcal{G} \rightarrow \mathcal{G}$ be a linear map satisfying the operator form of classical Yang-Baxter equation ([Se] and the Remark after Proposition 7.5)

$$
[R(x), R(y)]=R([R(x), y]+[x, R(y)]), \quad \forall x, y \in \mathcal{G}
$$

Then

$$
x * y=[R(x), y], \quad \forall x, y \in \mathcal{G}
$$

defines a left-symmetric algebra ([GS],[BM5]). Recall that the matrix form of the above $R$ (satisfying equation (2.7)) is a classical $r$-matrix. Hence the above construction of left-symmetric algebras can be regarded as a Lie algebra "left-twisted" by a classical $r$-matrix, which gives an algebraic interpretation of "left-symmetry" (comparing with the geometric interpretation given in the Introduction) ([Bai3]).

Example 2.3 Let $(A, \cdot)$ be an associative algebra and $R: A \rightarrow A$ be a linear map satisfying

$$
R(x) \cdot R(y)+R(x \cdot y)=R(R(x) \cdot y+x \cdot R(y)), \quad \forall x, y \in A .
$$

Then

$$
x * y=R(x) \cdot y-y \cdot R(x)-x \cdot y, \quad \forall x, y \in A
$$

defines a left-symmetric algebra ([GS]). The above $R$ is called a Rota-Baxter operator which was introduced to solve analytic $([\mathrm{Bax}])$ and combinatorial problems $([\mathrm{R}])$ and attracts more attention in many fields in mathematics and mathematical physics ([EGK] and the references therein). It is also related to the "modified classical Yang-Baxter equation" ([Se]).

Example 2.4 Let $V$ be a vector space over the complex field $\mathbf{C}$ with the ordinary scalar product $($,$) and a$ be a fixed vector in $V$, then

$$
u * v=(u, v) a+(u, a) v, \quad \forall u, v \in V
$$


defines a left-symmetric algebra on $V$ which gives the integrable (generalized) Burgers equation $([\mathrm{SS}])$

$$
U_{t}=U_{x x}+2 U * U_{x}+(U *(U * U))-((U * U) * U)
$$

In [Bai1], we generalized the above construction to get left-symmetric algebras from linear functions and in particular, we proved that the left-symmetric algebras given by equation (2.11) are simple (without any ideals except zero and itself) (cf. [Bu1]).

On the other hand,

Definition 2.2 A Lie algebra $\mathcal{G}$ is called a symplectic Lie algebra if there is a nondegenerate skew-symmetric 2 -cocycle $\omega$ (the symplectic form) on $\mathcal{G}$, that is,

$$
\omega([x, y], z)+\omega([y, z], x)+\omega([z, x], y)=0, \quad \forall x, y, z \in \mathcal{G}
$$

We denote it by $(\mathcal{G}, \omega)$. Let $\mathcal{H}$ be a subalgebra of a symplectic Lie algebra $(\mathcal{G}, \omega)$ and $\mathcal{H}^{\perp}=$ $\{x \in \mathcal{G} \mid \omega(x, y)=0, \forall y \in \mathcal{H}\}$. If $\mathcal{H} \subset \mathcal{H}^{\perp}$, then $\mathcal{H}$ is called totally isotropic. If $\mathcal{H}=\mathcal{H}^{\perp}$, then $\mathcal{H}$ is called Lagrangian.

Theorem $2.2([\mathrm{Ch}])$ Let $(\mathcal{G}, \omega)$ be a symplectic Lie algebra. Then there exists a compatible left-symmetric algebra structure " $*$ " on $\mathcal{G}$ given by

$$
\omega(x * y, z)=-\omega(y,[x, z]), \quad \forall x, y, z \in \mathcal{G} .
$$

Throughout this paper, we mainly study the following symplectic Lie algebras.

Definition 2.3 Let $(\mathcal{G}, \omega)$ be a symplectic Lie algebra. $\mathcal{G}$ is called a parakähler Lie algebra if $\mathcal{G}$ is a direct sum of the underlying vector spaces of two Lagrangian subalgebras $\mathcal{G}^{+}$and $\mathcal{G}^{-}$. It is denoted by $\left(\mathcal{G}, \mathcal{G}^{+}, \mathcal{G}^{-}, \omega\right)$. Two parakähler Lie algebras $\left(\mathcal{G}_{1}, \mathcal{G}_{1}^{+}, \mathcal{G}_{1}^{-}, \omega_{1}\right)$ and $\left(\mathcal{G}_{2}, \mathcal{G}_{2}^{+}, \mathcal{G}_{2}^{-}, \omega_{2}\right)$ are isomorphic if there exists a Lie algebra isomorphism $\varphi: \mathcal{G}_{1} \rightarrow \mathcal{G}_{2}$ such that

$$
\varphi\left(\mathcal{G}_{1}^{+}\right)=\mathcal{G}_{2}^{+}, \quad \varphi\left(\mathcal{G}_{1}^{-}\right)=\mathcal{G}_{2}^{-}, \omega_{1}(x, y)=\varphi^{*} \omega_{2}(x, y)=\omega_{2}(\varphi(x), \varphi(y)), \quad \forall x, y \in \mathcal{G}_{1}
$$

It is easy to see that a symplectic Lie algebra $(\mathcal{G}, \omega)$ is a parakähler Lie algebra if and only if $\mathcal{G}$ is a direct sum of the underlying vector spaces of two totally isotropic subalgebras.

Proposition 2.3 ([Bai2]) $\quad$ Let $\left(\mathcal{G}, \mathcal{G}^{+}, \mathcal{G}^{-}, \omega\right)$ be a parakähler Lie algebra, then there exists a left-symmetric algebra structure on $\mathcal{G}$ given by equation $(2.14)$ such that $\mathcal{G}^{+}$and $\mathcal{G}^{-}$are left-symmetric subalgebras. Moreover, two parakähler Lie algebras $\left(\mathcal{G}_{i}, \mathcal{G}_{i}^{+}, \mathcal{G}_{i}^{-}, \omega_{i}\right)(i=1,2)$ are isomorphic if and only if there exist an isomorphism of left-symmetric algebras satisfying equation (2.15) which the compatible left-symmetric algebras are given by equation (2.14). 
Definition 2.4 Let $\mathcal{G}$ be a Lie algebra. If there is a Lie algebra structure on a direct sum of the underlying vector spaces of $\mathcal{G}$ and its dual space $\mathcal{G}^{*}=\operatorname{Hom}(\mathcal{G}, \mathbf{F})$ such that $\mathcal{G}$ and $\mathcal{G}^{*}$ are Lie subalgebras and the natural skew-symmetric bilinear form on $\mathcal{G} \oplus \mathcal{G}^{*}$

$$
\omega_{p}\left(x+a^{*}, y+b^{*}\right)=\left\langle a^{*}, y\right\rangle-\left\langle x, b^{*}\right\rangle, \quad \forall x, y \in \mathcal{G}, a^{*}, b^{*} \in \mathcal{G}^{*}
$$

is a 2 -cocycle, where $\langle$,$\rangle is the ordinary pair between \mathcal{G}$ and $\mathcal{G}^{*}$, then it is called a phase space of the Lie algebra $\mathcal{G}([\mathrm{Ku} 1],[$ Bai2] $)$.

Obviously, a phase space of a Lie algebra is a parakähler Lie algebra. Furthermore, we have

Proposition 2.4 Every parakähler Lie algebra $\left(\mathcal{G}, \mathcal{G}^{+}, \mathcal{G}^{-}, \omega\right)$ is isomorphic to a phase space of $\mathcal{G}^{+}$.

Proof Let $\varphi: \mathcal{G}^{-} \rightarrow\left(\mathcal{G}^{+}\right)^{*}$ be a linear isomorphism given by $\langle\varphi(a), x\rangle=\omega(a, x)$ for any $x \in \mathcal{G}^{+}$and $a \in \mathcal{G}^{-}$. Extending $\varphi$ to be a linear isomorphism from $\mathcal{G}=\mathcal{G}^{+} \oplus \mathcal{G}^{-}$to $\mathcal{G}_{p}=\mathcal{G}^{+} \oplus\left(\mathcal{G}^{+}\right)^{*}$ with $\varphi(x)=x$ for any $x \in \mathcal{G}^{+}$. Let the Lie algebra structure on $\mathcal{G}_{p}$ given by

$$
[x, y]=[x, y],\left[a^{*}, b^{*}\right]=\varphi\left[\varphi^{-1}\left(a^{*}\right), \varphi^{-1}\left(b^{*}\right)\right], \quad\left[x, a^{*}\right]=\varphi\left(\left[x, \varphi^{-1}\left(a^{*}\right)\right]\right), \quad \forall x \in \mathcal{G}^{+}, a^{*} \in\left(\mathcal{G}^{+}\right)^{*}
$$

Therefore $\varphi$ is an isomorphism of Lie algebras. Furthermore, for any $x, y \in \mathcal{G}^{+}, a, b \in \mathcal{G}^{-}$, we have

$$
\omega(x+a, y+b)=\omega(a, y)+\omega(x, b)=\langle\varphi(a), y\rangle-\langle x, \varphi(b)\rangle=\omega_{p}(\varphi(x+a), \varphi(y+b)) .
$$

Therefore $\omega_{p}$ is a 2-cocycle in the above Lie algebra $\mathcal{G}_{p}$ and $\varphi$ is an isomorphism of parakähler Lie algebras.

Remark It is obvious that, by symmetry, every parakähler Lie algebra $\left(\mathcal{G}, \mathcal{G}^{+}, \mathcal{G}^{-}, \omega\right)$ is also isomorphic to a phase space of $\mathcal{G}^{-}$.

On the other hand, recall that $(\mathcal{G}, \mathcal{H}, \rho, \mu)$ is a matched pair of Lie algebras if $\mathcal{G}$ and $\mathcal{H}$ are Lie algebras and $\rho: \mathcal{G} \rightarrow g l(\mathcal{H})$ and $\mu: \mathcal{H} \rightarrow g l(\mathcal{G})$ are representations satisfying

$$
\begin{gathered}
\rho(x)[a, b]-[\rho(x) a, b]-[a, \rho(x) b]+\rho(\mu(a) x) b-\rho(\mu(b) x) a=0 \\
\mu(a)[x, y]-[\mu(a) x, y]-[x, \mu(a) y]+\mu(\rho(x) a) y-\mu(\rho(y) a) x=0,
\end{gathered}
$$

for any $x, y \in \mathcal{G}$ and $a, b \in \mathcal{H}$. In this case, there exists a Lie algebra structure on the vector space $\mathcal{G} \oplus \mathcal{H}$ given by

$$
[x+a, y+b]=[x, y]+\mu(a) y-\mu(b) x+[a, b]+\rho(x) b-\rho(y) a, \quad \forall x, y \in \mathcal{G}, a, b \in \mathcal{H}
$$


We denote it by $\mathcal{G} \bowtie_{\mu}^{\rho} \mathcal{H}$ or simply $\mathcal{G} \bowtie \mathcal{H}$. Moreover, every Lie algebra which is a direct sum of the underlying vector spaces of two subalgebras can be obtained from a matched pair of Lie algebras ([Maj], [T]).

Theorem 2.5 ([Bai2]) Let $(A, \cdot)$ be a left-symmetric algebra. Suppose there is another left-symmetric algebra structure "o" on its dual space $A^{*}$. Let $L^{*}$ and $L_{\circ}^{*}$ be the dual representation of the regular representation of $\mathcal{G}(A)$ and $\mathcal{G}\left(A^{*}\right)$ respectively, that is,

$$
\left\langle L^{*}(x) a^{*}, y\right\rangle=-\left\langle a^{*}, x \cdot y\right\rangle, \quad\left\langle L_{\circ}^{*}\left(a^{*}\right) x, b^{*}\right\rangle=-\left\langle x, a^{*} \circ b^{*}\right\rangle, \quad \forall x, y \in A, \quad a^{*}, b^{*} \in A^{*} .
$$

Then there exists a parakähler Lie algebra structure on the vector space $A \oplus A^{*}$ such that $\mathcal{G}(A)$ and $\mathcal{G}\left(A^{*}\right)$ are Lagrangian subalgebras associated to the symplectic form (2.16) if and only if $\left(\mathcal{G}(A), \mathcal{G}\left(A^{*}\right), L_{.}^{*}, L_{\circ}^{*}\right)$ is a matched pair of Lie algebras. Furthermore, every parakähler Lie algebra can be obtained from the above way.

\section{Bimodules and matched pairs of left-symmetric algebras}

Definition 3.1 Let $A$ be a left-symmetric algebra and $V$ be a vector space. Let $S, T: A \rightarrow$ $g l(V)$ be two linear maps. $V$ (or the pair $(S, T)$, or $(S, T, V)$ ) is called a bimodule of $A$ if

$$
\begin{gathered}
S(x) S(y) v-S(x y) v=S(y) S(x) v-S(y x) v, \quad \forall x, y \in A, v \in V \\
S(x) T(y) v-T(y) S(x) v=T(x y) v-T(y) T(x) v, \quad \forall x, y \in A, v \in V .
\end{gathered}
$$

According to $[\mathrm{Sc}]$, the following result is obvious.

Proposition 3.1 Let $(A, \cdot)$ be a left-symmetric algebra and $V$ be a vector space. Let $S, T: A \rightarrow g l(V)$ be two linear maps. Then $(S, T)$ is a bimodule of $A$ if and only if the direct sum $A \oplus V$ of vector spaces is turned into a left-symmetric algebra (the semidirect sum) by defining multiplication in $A \oplus V$ by

$$
\left(x_{1}+v_{1}\right) *\left(x_{2}+v_{2}\right)=x_{1} \cdot x_{2}+\left(S\left(x_{1}\right) v_{2}+T\left(x_{2}\right) v_{1}\right), \quad \forall x_{1}, x_{2} \in, v_{1}, v_{2} \in V
$$

We denote it by $A \ltimes_{S, T} V$ or simply $A \ltimes V$.

Lemma 3.2 Let $(S, T, V)$ be a bimodule of a left-symmetric algebra $A$. Then

(1) $S: A \rightarrow g l(V)$ is a representation of the sub-adjacent Lie algebra $\mathcal{G}(A)$.

(2) $\rho=S-T$ is a representation of the Lie algebra $\mathcal{G}(A)$.

(3) For any representation $\mu: \mathcal{G}(A) \rightarrow g l(\mathcal{G}(A))$ of the Lie algebra $\mathcal{G}(A),(\mu, 0)$ is a bimodule of $A$. 
(4) The left-symmetric algebras $A \ltimes_{S, T} V$ and $A \ltimes_{S-T, 0} V$ given by the bimodules $(S, T)$ and $(S-T, 0)$ respectively have the same sub-adjacent Lie algebra:

$$
\left[x_{1}+v_{1}, x_{2}+v_{2}\right]=\left[x_{1}, x_{2}\right]+(S-T)\left(x_{1}\right) v_{2}-(S-T)\left(x_{2}\right) v_{1}, \quad \forall x_{1}, x_{2} \in \mathcal{G}(A), v_{1}, v_{2} \in V
$$

It is the semidirect sum of a Lie algebra $\mathcal{G}(A)$ and its module $(\rho=S-T, V)$, we denote it by $\mathcal{G}(A) \ltimes_{S-T} V$.

Proof (1) and (3) follow directly from Definition 3.1. For any $x, y \in A$, we have

$$
\begin{aligned}
{[(S-T)(x),(S-T)(y)] } & =[S(x), S(y)]-[S(x), T(y)]-[T(x), S(y)]+[T(x), T(y)] \\
& =S([x, y])-T(x y)+T(y) T(x)+T(y x)-T(x) T(y)+[T(x), T(y)] \\
& =(S-T)([x, y]) .
\end{aligned}
$$

Thus $\rho=S-T$ is a representation of the Lie algebra $\mathcal{G}(A)$. Hence (4) follows immediately.

Proposition 3.3 Let $(A, \cdot)$ be a left-symmetric algebra and $(S, T, V)$ be its bimodule. Let $S^{*}, T^{*}: A \rightarrow g l\left(V^{*}\right)$ be the linear maps given by

$$
\left\langle S^{*}(x) u^{*}, v\right\rangle=-\left\langle S(x) v, u^{*}\right\rangle,\left\langle T^{*}(x) u^{*}, v\right\rangle=-\left\langle T(x) v, u^{*}\right\rangle, \forall x \in A, u^{*} \in V^{*}, v \in V .
$$

Then $\left(S^{*}-T^{*},-T^{*}, V^{*}\right)$ is a bimodule of $A$. Therefore there are two compatible left-symmetric algebra structures $A \ltimes_{S^{*}, 0} V^{*}$ and $A \ltimes_{S^{*}-T^{*},-T^{*}} V^{*}$ on the Lie algebra $\mathcal{G}(A) \ltimes_{S^{*}} V^{*}$.

Proof Obviously, $S^{*}-T^{*}$ is just the dual representation of the representation $\rho=S-T$ of the sub-adjacent Lie algebra $\mathcal{G}(A)$. For any $x, y \in A, u^{*} \in V^{*}, v \in V$, we have

$$
\begin{aligned}
& \left\langle\left[\left(S^{*}-T^{*}\right)(x),-T^{*}(y)\right] u^{*}, v\right\rangle=\left\langle\left(S^{*}-T^{*}\right)(x) u^{*},-T(y) v\right\rangle+\left\langle T^{*}(y) u^{*},(S-T)(x) v\right\rangle \\
& =\left\langle u^{*},(S-T)(x) T(y) v\right\rangle-\left\langle u^{*}, T(y)(S-T)(x) v\right\rangle=\left\langle u^{*},(T(x y)-T(x) T(y)) v\right\rangle \\
& =\left\langle-T^{*}(x y) u^{*}-T^{*}(y) T^{*}(x) u^{*}, v\right\rangle .
\end{aligned}
$$

Therefore $\left(S^{*}-T^{*},-T^{*}, V^{*}\right)$ is a bimodule of $A$. The other conclusions follow from Lemma 3.2 directly.

However, $\left(S^{*}, T^{*}, V^{*}\right)$ is not a bimodule of $A$ in general. Similar to the above discussion, we have the following conclusion:

Proposition 3.4 Let $A$ be a left-symmetric algebra and $(S, T, V)$ be its bimodule. Then the following conditions are equivalent:

(1) $(S-T,-T)$ is a bimodule of $A$;

(2) $\left(S^{*}, T^{*}\right)$ is a bimodule of $A$; 
(3) $T(x) T(y)=T(y) T(x)$ for any $x, y \in A$.

Example 3.1 Let $A$ be a left-symmetric algebra. Then $(L, 0),(\mathrm{ad}, 0)$ and $(L, R)$ are bimodules of $A$, where ad $=L-R: A \rightarrow g l(A)$ is the adjoint representation of the Lie algebra $\mathcal{G}(A)$. On the other hand, $\left(L^{*}, 0\right),\left(\mathrm{ad}^{*}, 0\right)$ and $\left(\mathrm{ad}^{*},-R^{*}\right)$ are bimodules of $A$, too, where $\mathrm{ad}^{*}$ and $L^{*}$ are the dual representations of the adjoint representation and the regular representation of the sub-adjacent Lie algebra $\mathcal{G}(A)$ respectively, and $R^{*}=-\mathrm{ad}^{*}+L^{*}$.

Example 3.2 Let $A$ be a left-symmetric algebra. Then $\left(L^{*}, R^{*}\right)$ is a bimodule of $A$ if and only if $(\mathrm{ad},-R)$ is a bimodule of $A$, if and only if $A$ is a Novikov algebra.

Example 3.3 Let $A$ be a left-symmetric algebra. Then there are two compatible leftsymmetric algebras $A \ltimes_{L^{*}, 0} A^{*}$ and $A \ltimes_{\mathrm{ad}^{*},-R^{*}} A^{*}$ on the Lie algebra $\mathcal{G}(A) \ltimes_{L^{*}} \mathcal{G}^{*}(A)$, which are given by the bimodules $\left(L^{*}, 0\right)$ and $\left(\mathrm{ad}^{*},-R^{*}\right)$ respectively. This conclusion has already been given in [Bai2].

Next, we study how to construct a left-symmetric algebra structure on a direct $\operatorname{sum} A \oplus B$ of the underlying vector spaces of two left-symmetric algebras $A$ and $B$ such that $A$ and $B$ are subalgebras.

Theorem 3.5 Let $(A, \cdot)$ and $(B, \circ)$ be two left-symmetric algebras. Suppose that there are linear maps $l_{A}, r_{A}: A \rightarrow g l(B)$ and $l_{B}, r_{B}: B \rightarrow g l(A)$ such that $\left(l_{A}, r_{A}\right)$ is a bimodule of $A$ and $\left(l_{B}, r_{B}\right)$ is a bimodule of $B$ and they satisfy the following conditions:

$$
\begin{gathered}
r_{A}(x)[a, b]=r_{A}\left(l_{B}(b) x\right) a-r_{A}\left(l_{B}(a) x\right) b+a \circ\left(r_{A}(x) b\right)-b \circ\left(r_{A}(x) a\right) \\
l_{A}(x)(a \circ b)=-l_{A}\left(l_{B}(a) x-r_{B}(a) x\right) b+\left(l_{A}(x) a-r_{A}(x) a\right) \circ b+r_{A}\left(r_{B}(b) x\right) a+a \circ\left(l_{A}(x) b\right) \\
r_{B}(a)[x, y]=r_{B}\left(l_{A}(y) a\right) x-r_{B}\left(l_{A}(x) a\right) y+x \cdot\left(r_{B}(a) y\right)-y \cdot\left(r_{B}(a) x\right) \\
l_{B}(a)(x \cdot y)=-l_{B}\left(l_{A}(x) a-r_{A}(x) a\right) y+\left(l_{B}(a) x-r_{B}(a) x\right) \cdot y+r_{B}\left(r_{A}(y) a\right) x+x \cdot\left(l_{B}(a) y\right)
\end{gathered}
$$

for any $x, y \in A, a, b \in B$. Then there is a left-symmetric algebra structure on the vector space $A \oplus B$ given by

$$
(x+a) *(y+b)=\left(x \cdot y+l_{B}(a) y+r_{B}(b) x\right)+\left(a \circ b+l_{A}(x) b+r_{A}(y) a\right), \quad \forall x, y \in A, a, b \in B .
$$

We denote this left-symmetric algebra by $A \bowtie_{l_{B}, r_{B}}^{l_{A}, r_{A}} B$ or simply $A \bowtie B$. And $\left(A, B, l_{A}, r_{A}, l_{B}, r_{B}\right)$ satisfying the above conditions is called a matched pair of left-symmetric algebras. On the other hand, every left-symmetric algebra which is a direct sum of the underlying vector spaces of two subalgebras can be obtained from the above way. 
Proof For any $x, y, z \in A$ and $a, b, c \in B$, the associator in $A \bowtie B$ satisfies

$(x+a, y+b, z+c)=(x, y, z)+(x, b, c)+(a, y, c)+(x, y, c)+(x, b, z)+(a, b, c)+(a, y, z)+(a, b, z)$.

Therefore, the equation (3.10) defines a left-symmetric algebra structure on $A \oplus B$ if and only if the following equations are satisfied:

$$
\begin{aligned}
& (x, y, z)=(y, x, z) \Longleftrightarrow A \text { is a left - symmetric algebra; } \\
& (x, y, c)=(y, x, c) \quad \Longleftrightarrow \quad l_{A} \text { is a representation of } \mathcal{G}(A) \text { and equation (3.8) holds; } \\
& (a, y, z)=(y, a, z) \Longleftrightarrow[(x, b, z)=(b, x, z) \Longleftrightarrow] \text { equation (3.9) holds and } r_{A} \text { satisfies } \\
& l_{A}(y) r_{A}(z) a-r_{A}(z) l_{A}(y) a=r_{A}(y \cdot z) a-r_{A}(z) r_{A}(y) a \\
& (a, b, c)=(b, a, c) \quad \Longleftrightarrow \quad B \text { is a left - symmetric algebra; } \\
& (a, b, z)=(b, a, z) \quad \Longleftrightarrow \quad l_{B} \text { is a representation of } \mathcal{G}(B) \text { and equation (3.6) holds; } \\
& (x, b, c)=(b, x, c) \Longleftrightarrow[(a, y, c)=(y, a, c) \Longleftrightarrow] \text { equation }(3.7) \text { holds and } r_{B} \text { satisfies } \\
& l_{B}(b) r_{B}(c) x-r_{B}(c) l_{B}(b) x=r_{B}(b \circ c) x-r_{B}(c) r_{B}(b) x .
\end{aligned}
$$

Hence $A \bowtie B$ is a left-symmetric algebra if and only if $\left(l_{A}, r_{A}\right)$ is a bimodule of $A$ and $\left(l_{B}, r_{B}\right)$ is a bimodule of $B$ and equations (3.6-3.9) hold. On the other hand, if $A$ and $B$ are left-symmetric subalgebras of a left-symmetric $C$ such that $C=A \oplus B$ which is a direct sum of the underlying vector spaces of $A$ and $B$, then it is easy to show that the linear maps $l_{A}, r_{A}: A \rightarrow g l(B)$ and $l_{B}, r_{B}: B \rightarrow g l(B)$ determined by

$$
x * a=l_{A}(x) a+r_{B}(a) x, a * x=l_{B}(a) x+r_{A}(x) a, \quad \forall x \in A, a \in B
$$

satisfy equations (3.6-3.9). In addition, $\left(l_{A}, r_{A}\right)$ is a bimodule of $A$ and $\left(l_{B}, r_{B}\right)$ is a bimodule of $B$.

Remark Obviously $B$ is an ideal of $A \bowtie B$ if and only if $l_{B}=r_{B}=0$. In this case, if in addition, $B$ is trivial (that is, all the products of $B$ are zero), then $A \bowtie_{0,0}^{l_{A}, r_{A}} B \cong A \ltimes_{l_{A}, r_{A}} B$. Furthermore, some other special cases of the above theorem have already been studied. For example, Diatta and Medina studied the case that both $A$ and $B$ are left ideals of $A \bowtie B$, that is, $r_{A}=r_{B}=0([\mathrm{DiM}])$.

Corollary 3.6 ([DiM, Lemma 3.0.3]) Let $(A, \cdot)$ and $(B, \circ)$ be two left-symmetric algebras. Suppose there exist two Lie algebra representations $\rho: \mathcal{G}(A) \rightarrow g l(B)$ and $\mu: \mathcal{G}(B) \rightarrow g l(A)$ such that

$$
\rho(x)(a \circ b)=\rho(x) a \circ b+a \circ(\rho(x) b)-\rho(\mu(a) x) b ;
$$




$$
\mu(a)(x \cdot y)=\mu(a) x \cdot y+x \cdot(\mu(a) y)-\mu(\rho(x) a) y,
$$

for any $x, y \in A, a, b \in B$. $A$ and $B$ are called to be $(\rho, \mu)$-linked. Then there is a left-symmetric algebra structure on the vector space $A \oplus B$ given by

$$
(x+a) *(y+b)=(x \cdot y+\mu(a) y)+(a \circ b+\rho(x) b), \forall x, y \in A, a, b \in B .
$$

Therefore, its sub-adjacent Lie algebra (which is the same Lie algebra given by the matched pair of Lie algebras $(\mathcal{G}(A), \mathcal{G}(B), \rho, \mu))$ is given by

$[(x+a),(y+b)]=(x \cdot y-y \cdot x+\mu(a) y-\mu(b) x)+(a \circ b-b \circ a+\rho(x) b-\rho(y) a), \forall x, y \in A, a, b \in B$.

Corollary 3.7 Let $\left(A, B, l_{A}, r_{A}, l_{B}, r_{B}\right)$ be a matched pair of left-symmetric algebras. Then $\left(\mathcal{G}(A), \mathcal{G}(B), l_{A}-r_{A}, l_{B}-r_{B}\right)$ is a matched pair of Lie algebras.

Proof This conclusion can be proved by a direct computation or from the relation between the left-symmetric algebra $A \bowtie B$ and its sub-adjacent algebra Lie algebra. In fact, the sub-adjacent Lie algebra $\mathcal{G}(A \bowtie B)$ is just the Lie algebra obtained from the matched pair $(\mathcal{G}(A), \mathcal{G}(B), \rho, \mu)$ :

$$
[x+a, y+b]=[x, y]+\mu(a) y-\mu(b) x+[a, b]+\rho(x) b-\rho(y) a, \quad \forall x, y \in \mathcal{G}(A), a, b \in \mathcal{G}(B),
$$

where $\rho=l_{A}-r_{A}, \mu=l_{B}-r_{B}$.

Now we turns to the case of a parakähler Lie algebra: $\rho=L^{*}$ and $\mu=L_{\circ}^{*}$.

Theorem 3.8 Let $(A, \cdot)$ be a left-symmetric algebra. Suppose there is another leftsymmetric algebra structure "o" on its dual space $A^{*}$. Then $\left(\mathcal{G}(A), \mathcal{G}\left(A^{*}\right), L_{.}^{*}, L_{\circ}^{*}\right)$ is a matched pair of Lie algebras if and only if $\left(A, A^{*}, \mathrm{ad}^{*},-R_{.}^{*}, \mathrm{ad}_{\circ}^{*},-R_{\circ}^{*}\right)$ is a matched pair of left-symmetric algebras.

Proof We only need to prove the "only if" part. A direct proof is given as follows. In fact, in the case $l_{A}=\mathrm{ad}^{*}, r_{A}=-R^{*}, l_{B}=l_{A^{*}}=\operatorname{ad}_{\circ}^{*}, r_{B}=r_{A^{*}}=-R_{\circ}^{*}, \rho=L^{*}$ and $\mu=L_{\circ}^{*}$, we have

$$
\begin{aligned}
& \text { equation }(2.17) \Longleftrightarrow \text { equation (3.6) } \Longleftrightarrow \text { equation (3.9); } \\
& \text { equation }(2.18) \Longleftrightarrow \text { equation (3.7) } \Longleftrightarrow \text { equation (3.8). }
\end{aligned}
$$

As an example (the proof of other equivalent relations is similar), we show how equation (2.17) is equivalent to equation (3.6). In fact, it follows from

$$
\left\langle-R_{.}^{*}(x)\left[a^{*}, b^{*}\right], y\right\rangle=-\left\langle L^{*}(y)\left[a^{*}, b^{*}\right], x\right\rangle
$$




$$
\begin{aligned}
& \left\langle-R_{.}^{*}\left(\operatorname{ad}_{\circ}^{*}\left(b^{*}\right) x\right) a^{*}, y\right\rangle=\left\langle\left[b^{*}, L_{.}^{*}(y) a^{*}\right], x\right\rangle ; \\
& -\left\langle-R_{\cdot}^{*}\left(\operatorname{ad}_{\circ}^{*}\left(a^{*}\right) x\right) b^{*}, y\right\rangle=\left\langle-\left[a^{*}, L_{.}^{*}(y) b^{*}\right], x\right\rangle ; \\
& \left\langle a^{*} \circ\left(-R_{.}^{*}(x) b^{*}\right), y\right\rangle=\left\langle L_{.}^{*}\left(L_{\circ}^{*}\left(a^{*}\right) y\right)\left(b^{*}\right), x\right\rangle ; \\
& \left\langle-b^{*} \circ\left(-R_{\cdot}^{*}(x) a^{*}\right), y\right\rangle=\left\langle-L_{.}^{*}\left(L_{\circ}^{*}\left(b^{*}\right) y\right)\left(a^{*}\right), x\right\rangle,
\end{aligned}
$$

for any $x, y \in A$ and $a^{*}, b^{*} \in A^{*}$.

There is another proof. If $\left(\mathcal{G}(A), \mathcal{G}\left(A^{*}\right), L^{*}, L_{\circ}^{*}\right)$ is a matched pair of Lie algebras, then $\mathcal{G}(A) \bowtie_{L_{\circ}^{*}}^{L^{*}} \mathcal{G}\left(A^{*}\right)$ is a parakähler Lie algebra with the natural symplectic form (2.16). Hence there exists a compatible left-symmetric algebra structure on $\mathcal{G}(A) \bowtie_{L_{\circ}^{*}}^{L_{*}^{*}} \mathcal{G}\left(A^{*}\right)$ given by equation (2.14). With a simple and direct computation, we know that $A$ and $A^{*}$ are its subalgebras and the other products are given by

$$
x * a^{*}=\operatorname{ad}_{.}^{*}(x) a^{*}-R_{\circ}^{*}\left(a^{*}\right)(x), a^{*} * x=\operatorname{ad}_{\circ}^{*}\left(a^{*}\right) x-R_{.}^{*}(x) a^{*}, \forall x \in A, a^{*} \in A^{*} .
$$

Therefore $\left(A, A^{*}\right.$, ad. $^{*},-R_{.}^{*}$, ad $\left._{\circ}^{*},-R_{\circ}^{*}\right)$ is a matched pair of left-symmetric algebras.

Remark Unlike the case of semidirect sum in Example 3.3, it is not true that there is a compatible left-symmetric structure on the Lie algebra $\mathcal{G}(A) \bowtie_{L_{\circ}^{*}}^{L^{*}} \mathcal{G}\left(A^{*}\right)$ coming from the bimodules $\left(L_{.}^{*}, 0\right)$ and $\left(L_{\circ}^{*}, 0\right)$ since in this case, $\left(A, A^{*}, L_{.}^{*}, 0, L_{\circ}^{*}, 0\right)$ is not a matched pair of left-symmetric algebras in general. In fact, from Corollary $3.6,\left(A, A^{*}, L_{.}^{*}, 0, L_{\circ}^{*}, 0\right)$ is a matched pair of left-symmetric algebras if and only if

$$
\begin{gathered}
L_{.}^{*}(x)\left(a^{*} \circ b^{*}\right)=-L_{.}^{*}\left(L_{\circ}^{*}\left(a^{*}\right) x\right) b^{*}+L_{.}^{*}(x) a^{*} \circ b^{*}+a^{*} \circ L_{.}^{*}(x) b^{*} ; \\
L_{\circ}^{*}\left(a^{*}\right)(x \cdot y)=-L_{\circ}^{*}\left(L_{.}^{*}(x) a^{*}\right) y+L_{\circ}^{*}\left(a^{*}\right) x \cdot y+x \cdot L_{\circ}^{*}\left(a^{*}\right) y,
\end{gathered}
$$

for any $x, y \in A$ and $a^{*}, b^{*} \in A^{*}$ which is a little stronger than equations (2.17-2.18) in the case $\rho=L^{*}$ and $\mu=L_{\circ}^{*}$.

\section{Left-symmetric bialgebras}

At first, we give some notations as follows.

Let $\mathcal{G}$ be a Lie algebra. For any two representations $(\rho, V)$ and $(\mu, W)$ of $\mathcal{G}$, it is easy to know that $(\rho \otimes 1+1 \otimes \mu, V \otimes W)$ is also a representation of $\mathcal{G}$, where

$$
(\rho \otimes 1+1 \otimes \mu)(x)(v \otimes w)=(\rho(x) \otimes 1+1 \otimes \mu(x))(v \otimes w)=\rho(x) v \otimes w+v \otimes \mu(x) w
$$

for any $x \in \mathcal{G}, v \in V, w \in W$. 
For a Lie algebra $\mathcal{G}$ and a representation $(\rho, V)$ of $\mathcal{G}$, recall that a 1-cocycle $\delta$ associated to $\rho($ denoted by $(\rho, \delta))$ is a linear map from $\mathcal{G}$ to $V$ satisfying

$$
\delta([x, y])=\rho(x) \delta(y)-\rho(y) \delta(x), \quad \forall x, y \in \mathcal{G} .
$$

For a linear map $\phi: V_{1} \rightarrow V_{2}$, we denote the dual (linear) map by $\phi^{*}: V_{2}^{*} \rightarrow V_{1}^{*}$ given by

$$
\left\langle v, \phi^{*}\left(u^{*}\right)\right\rangle=\left\langle\phi(v), u^{*}\right\rangle, \quad \forall v \in V_{1}, u^{*} \in V_{2} .
$$

Theorem 4.1 Let $(A, \cdot)$ be a left-symmetric algebra whose product is given by a linear map $\beta^{*}: A \otimes A \rightarrow A$. Suppose there is another left-symmetric algebra structure "o" on its dual space $A^{*}$ given by a linear map $\alpha^{*}: A^{*} \otimes A^{*} \rightarrow A^{*}$. Then $\left(\mathcal{G}(A), \mathcal{G}\left(A^{*}\right), L_{.}^{*}, L_{\circ}^{*}\right)$ is a matched pair of Lie algebras if and only if $\alpha: A \rightarrow A \otimes A$ is a 1-cocycle of $\mathcal{G}(A)$ associated to $L . \otimes 1+1 \otimes$ ad. and $\beta: A^{*} \rightarrow A^{*} \otimes A^{*}$ is a 1-cocycle of $\mathcal{G}\left(A^{*}\right)$ associated to $L_{\circ} \otimes 1+1 \otimes \operatorname{ad}_{\circ}$.

Proof Let $\left\{e_{1}, \cdots, e_{n}\right\}$ be a basis of $A$ and $\left\{e_{1}^{*}, \cdots, e_{n}^{*}\right\}$ be its dual basis. Set $e_{i} \cdot e_{j}=$ $\sum_{k=1}^{n} c_{i j}^{k} e_{k}$ and $e_{i}^{*} \circ e_{j}^{*}=\sum_{k=1}^{n} f_{i j}^{k} e_{k}^{*}$. Therefore, we have

$\alpha\left(e_{k}\right)=\sum_{i, j=1}^{n} f_{i j}^{k} e_{i} \otimes e_{j}, \quad \beta\left(e_{k}^{*}\right)=\sum_{i, j=1}^{n} c_{i j}^{k} e_{i}^{*} \otimes e_{j}^{*} ; \quad L_{.}^{*}\left(e_{i}\right) e_{j}^{*}=-\sum_{k=1}^{n} c_{i k}^{j} e_{k}^{*}, \quad L_{\circ}^{*}\left(e_{i}^{*}\right) e_{j}=-\sum_{k=1}^{n} f_{i k}^{j} e_{k}$.

Hence the coefficient of $e_{m} \otimes e_{n}$ in

$$
\alpha\left(\left[e_{i}, e_{j}\right]\right)=\left[L .\left(e_{i}\right) \otimes 1+1 \otimes \operatorname{ad} .\left(e_{i}\right)\right] \alpha\left(e_{j}\right)-\left[L .\left(e_{j}\right) \otimes 1+1 \otimes \operatorname{ad} .\left(e_{j}\right)\right] \alpha\left(e_{i}\right)
$$

gives the following relation (for any $i, j, m, n$ )

$$
\sum_{k=1}^{n}\left(c_{i j}^{k}-c_{j i}^{k}\right) f_{m n}^{k}=\sum_{k=1}^{n}\left[c_{i k}^{m} f_{k n}^{j}+\left(c_{i k}^{n}-c_{k i}^{n}\right) f_{m k}^{j}-c_{j k}^{m} f_{k n}^{i}-\left(c_{j k}^{n}-c_{k j}^{n}\right) f_{m k}^{i}\right],
$$

which is precisely the relation given by the coefficient of $e_{n}$ in

$$
-L_{\circ}^{*}\left(e_{m}^{*}\right)\left[e_{i}, e_{j}\right]=L_{\circ}^{*}\left(L_{.}^{*}\left(e_{i}\right) e_{m}^{*}\right) e_{j}-\left[e_{i}, L_{\circ}^{*}\left(e_{m}^{*}\right) e_{j}\right]-L_{\circ}^{*}\left(L_{.}^{*}\left(e_{j}\right) e_{m}^{*}\right) e_{i}-\left[L_{\circ}^{*}\left(e_{m}^{*}\right) e_{i}, e_{j}\right] .
$$

Then $\alpha$ is a 1-cocycle of $\mathcal{G}(A)$ associated to $L . \otimes 1+1 \otimes$ ad. if and only if equation (2.18) holds in the case $\rho=L^{*}$. and $\mu=L_{\circ}^{*}$. Similarly (or by symmetry), $\beta$ is a 1-cocycle of $\mathcal{G}\left(A^{*}\right)$ associated to $L_{\circ} \otimes 1+1 \otimes \operatorname{ad}_{\circ}$ if and only if equation (2.17) holds.

Definition 4.1 Let $A$ be a vector space. A left-symmetric bialgebra structure on $A$ is a pair of linear maps $(\alpha, \beta)$ such that $\alpha: A \rightarrow A \otimes A, \beta: A^{*} \rightarrow A^{*} \otimes A^{*}$ and

(a) $\alpha^{*}: A^{*} \otimes A^{*} \rightarrow A^{*}$ is a left-symmetric algebra structure on $A^{*}$; 
(b) $\beta^{*}: A \otimes A \rightarrow A$ is a left-symmetric algebra structure on $A$;

(c) $\alpha$ is a 1 -cocycle of $\mathcal{G}(A)$ associated to $L \otimes 1+1 \otimes$ ad with values in $A \otimes A$;

(d) $\beta$ is a 1-cocycle of $\mathcal{G}\left(A^{*}\right)$ associated to $L \otimes 1+1 \otimes$ ad with values in $A^{*} \otimes A^{*}$.

We also denote this left-symmetric bialgebras by $\left(A, A^{*}, \alpha, \beta\right)$ or simply $\left(A, A^{*}\right)$.

Proposition 4.2 Let $(A, \cdot)$ be a left-symmetric algebra and $\left(A^{*}, \circ\right)$ be a left-symmetric algebra structure on its dual space $A^{*}$. Then the following conditions are equivalent:

(1) $\left(\mathcal{G}(A) \bowtie \mathcal{G}(A)^{*}, \mathcal{G}(A), \mathcal{G}\left(A^{*}\right), \omega_{p}\right)$ is a parakähler Lie algebra, where $\omega_{p}$ is given by equation (2.16);

(2) $\left(\mathcal{G}(A), \mathcal{G}\left(A^{*}\right), L_{.}^{*}, L_{\circ}^{*}\right)$ is a matched pair of Lie algebras;

(3) $\left(A, A^{*}, \operatorname{ad}^{*},-R_{.}^{*}, \operatorname{ad}_{\circ}^{*},-R_{\circ}^{*}\right)$ is a matched pair of left-symmetric algebras;

(4) $\left(A, A^{*}\right)$ is a left-symmetric bialgebra.

Proof The proofs are straightforward.

Definition 4.2 Let $\left(A, A^{*}, \alpha_{A}, \beta_{A}\right)$ and $\left(B, B^{*}, \alpha_{B}, \beta_{B}\right)$ be two left-symmetric bialgebras. A homomorphism of left-symmetric bialgebras $\varphi: A \rightarrow B$ is a homomorphism of left-symmetric algebras such that $\varphi^{*}: B^{*} \rightarrow A^{*}$ is also a homomorphism of left-symmetric algebras, that is, $\varphi$ satisfies

$$
(\varphi \otimes \varphi) \alpha_{A}(x)=\alpha_{B}(\varphi(x)), \quad\left(\varphi^{*} \otimes \varphi^{*}\right) \beta_{B}\left(a^{*}\right)=\beta_{A}\left(\varphi^{*}\left(a^{*}\right)\right), \forall x \in A, a^{*} \in B^{*} .
$$

An isomorphism of left-symmetric bialgebras is an invertible homomorphism of left-symmetric bialgebras.

Proposition 4.3 Two parakähler Lie algebras are isomorphic if and only if their corresponding left-symmetric bialgebras are isomorphic.

Proof Let $\left(\mathcal{G}(A) \bowtie \mathcal{G}\left(A^{*}\right), \mathcal{G}(A), \mathcal{G}\left(A^{*}\right), \omega_{p}\right)$ and $\left(\mathcal{G}(B) \bowtie \mathcal{G}\left(B^{*}\right), \mathcal{G}(B), \mathcal{G}\left(B^{*}\right), \omega_{p}\right)$ be two parakähler Lie algebras. Let $\left\{e_{1}, \cdots, e_{n}\right\}$ be a basis of $A$ and $\left\{e_{1}^{*}, \cdots, e_{n}^{*}\right\}$ be its dual basis. If $\varphi: \mathcal{G}(A) \bowtie \mathcal{G}\left(A^{*}\right) \rightarrow \mathcal{G}(B) \bowtie \mathcal{G}\left(B^{*}\right)$ is an isomorphism of parakähler Lie algebras, then $\left.\varphi\right|_{A}: A \rightarrow B$ and $\left.\varphi\right|_{A^{*}}: A^{*} \rightarrow B^{*}$ are isomorphisms of left-symmetric algebras due to Proposition 2.3. Moreover, $\left.\varphi\right|_{A^{*}}=\left(\left.\varphi\right|_{A}\right)^{*-1}$ since

$$
\begin{aligned}
\left\langle\left.\varphi\right|_{A^{*}}\left(e_{i}^{*}\right), \varphi\left(e_{j}\right)\right\rangle & =\omega_{p}\left(\left.\varphi\right|_{A^{*}}\left(e_{i}^{*}\right), \varphi\left(e_{j}\right)\right)=\omega_{p}\left(e_{i}^{*}, e_{j}\right) \\
& =\delta_{i j}=\left\langle e_{i}^{*}, e_{j}\right\rangle=\left\langle\varphi^{*}\left(\left.\varphi\right|_{A}\right)^{*-1}\left(e_{i}^{*}\right), e_{j}\right\rangle \\
& =\left\langle\left(\left.\varphi\right|_{A}\right)^{*-1}\left(e_{i}^{*}\right), \varphi\left(e_{j}\right)\right\rangle .
\end{aligned}
$$

Hence $\left(A, A^{*}\right)$ and $\left(B, B^{*}\right)$ are isomorphic as left-symmetric bialgebras. On the other hand, let $\left(A, A^{*}\right)$ and $\left(B, B^{*}\right)$ be two isomorphic left-symmetric bialgebras and $\varphi^{\prime}: A \rightarrow B$ be an 
isomorphism of left-symmetric bialgebras. Set $\varphi: A \oplus A^{*} \rightarrow B \oplus B^{*}$ be a linear map given by

$$
\varphi(x)=\varphi^{\prime}(x), \varphi\left(a^{*}\right)=\left(\varphi^{*}\right)^{-1}\left(a^{*}\right), \quad \forall x \in A, a^{*} \in A^{*} .
$$

Then it is easy to know that $\varphi$ is an isomorphism of the two parakähler Lie algebras $(\mathcal{G}(A) \bowtie$ $\left.\mathcal{G}\left(A^{*}\right), \mathcal{G}(A), \mathcal{G}\left(A^{*}\right), \omega_{p}\right)$ and $\left(\mathcal{G}(B) \bowtie \mathcal{G}\left(B^{*}\right), \mathcal{G}(B), \mathcal{G}\left(B^{*}\right), \omega_{p}\right)$.

Example 4.1 Let $\left(A, A^{*}, \alpha, \beta\right)$ be a left-symmetric bialgebra. Then its dual $\left(A^{*}, A, \beta, \alpha\right)$ is also a left-symmetric bialgebra.

Example 4.2 Let $A$ be a left-symmetric algebra. Let the left-symmetric algebra structure on $A^{*}$ be trivial, then in this case $\left(A, A^{*}, 0, \beta\right)$ is a left-symmetric bialgebra. And its corresponding left-symmetric algebra is $A \ltimes_{\mathrm{ad}^{*},-R^{*}} A^{*}$. Moreover, its corresponding parakähler Lie algebra is just the semidirect $\operatorname{sum} \mathcal{G}(A) \ltimes_{L^{*}} \mathcal{G}\left(A^{*}\right)$ with the symplectic form $\omega_{p}$ given by equation (2.16). Dually, let $A$ be a trivial left-symmetric algebra, then the left-symmetric bialgebra structures on $A$ are in one-to-one correspondence with the left-symmetric algebra structure on $A^{*}$.

Example 4.3 Let $\left(A, A^{*}\right)$ be a left-symmetric bialgebra. In next section, we will prove that there exists a natural left-symmetric bialgebra structure on the direct sum $A \oplus A^{*}$ of the underlying vector spaces of $A$ and $A^{*}$.

\section{$5 \quad$ Coboundary left-symmetric bialgebras}

In this section, we study the case that $\alpha$ is a 1-coboundary associated to $L \otimes 1+1 \otimes$ ad.

Definition 5.1 A left-symmetric bialgebra $\left(A, A^{*}, \alpha, \beta\right)$ is called coboundary if $\alpha$ is a 1coboundary of $\mathcal{G}(A)$ associated to $L \otimes 1+1 \otimes \mathrm{ad}$, that is, there exists a $r \in A \otimes A$ such that

$$
\alpha(x)=\left(L_{x} \otimes 1+1 \otimes \operatorname{ad} x\right) r, \quad \forall x \in A
$$

Let $A$ be a left-symmetric algebra whose product is given by $\beta^{*}: A \otimes A \rightarrow A$ and $r \in A \otimes A$. Suppose $\alpha: A \rightarrow A \otimes A$ is a 1-coboundary of $\mathcal{G}(A)$ associated to $L \otimes 1+1 \otimes$ ad (given by equation (5.1)). Then it is obvious that $\alpha$ is a 1-cocycle of $\mathcal{G}(A)$ associated to $L \otimes 1+1 \otimes$ ad. Therefore, $\left(A, A^{*}, \alpha, \beta\right)$ is a left-symmetric bialgebra if and only if the following two conditions are satisfied:

(1) $\alpha^{*}: A^{*} \otimes A^{*} \rightarrow A^{*}$ defines a left-symmetric algebra structure on $A^{*}$.

(2) $\beta$ is a 1-cocycle of $\mathcal{G}\left(A^{*}\right)$ associated to $L \otimes 1+1 \otimes \mathrm{ad}$, where the left-symmetric algebra structure of $A^{*}$ is given by (1). 
For any vector space $A$, let $\sigma: A \otimes A \rightarrow A \otimes A$ be the linear map (exchanging operator) satisfying $\sigma(x \otimes y)=y \otimes x$, for any $x, y \in A$. Let $r=\sum_{i} a_{i} \otimes b_{i} \in A \otimes A$, where $a_{i}, b_{i} \in A$. We denote $r_{12}=r$ and $r_{21}=\sigma(r)=\sum_{i} b_{i} \otimes a_{i}$.

Proposition 5.1 Let $(A, \cdot)$ be a left-symmetric algebra whose product is given by $\beta^{*}$ : $A \otimes A \rightarrow A$ and $r \in A \otimes A$. Suppose there exists a left-symmetric algebra structure "०" on $A^{*}$ given by $\alpha^{*}: A^{*} \otimes A^{*} \rightarrow A^{*}$, where $\alpha$ is given by equation (5.1). Then $\beta: A^{*} \rightarrow A^{*} \otimes A^{*}$ is a 1-cocycle of $\mathcal{G}\left(A^{*}\right)$ associated to $L \otimes 1+1 \otimes$ ad if and only if $r$ satisfies

$$
[P(x \cdot y)-P(x) P(y)]\left(r_{12}-r_{21}\right)=0, \forall x, y \in A,
$$

where $P(x)=L_{x} \otimes 1+1 \otimes L_{x}$.

Proof Let $\left\{e_{1}, \cdots, e_{n}\right\}$ be a basis of $A$ and $\left\{e_{1}^{*}, \cdots, e_{n}^{*}\right\}$ be its dual basis. Set $r=\sum_{i, j} a_{i j} e_{i} \otimes$ $e_{j}$. Suppose $e_{i} \cdot e_{j}=\sum_{k} c_{i j}^{k} e_{k}$ and $e_{i}^{*} \circ e_{j}^{*}=\sum_{k} f_{i j}^{k} e_{k}^{*}$. Since

$$
\alpha\left(e_{i}\right)=\sum_{k, l} f_{k l}^{i} e_{k} \otimes e_{l}=\left(L_{e_{i}} \otimes 1+1 \otimes \mathrm{ad} e_{i}\right) r
$$

we have (for any $i, k, l)$

$$
f_{k l}^{i}=\sum_{t}\left[a_{t l} c_{i t}^{k}+a_{k t}\left(c_{i t}^{l}-c_{t i}^{l}\right)\right]
$$

Therefore $\beta$ is a 1-cocycle of $\mathcal{G}\left(A^{*}\right)$ associated to $L_{\circ} \otimes 1+1 \otimes \operatorname{ad}_{\circ}$ if and only if

$$
\beta\left[e_{i}^{*}, e_{j}^{*}\right]=\left(L_{e_{i}^{*}} \otimes 1+1 \otimes \operatorname{ad} e_{i}^{*}\right) \beta^{*}\left(e_{j}^{*}\right)-\left(L_{e_{j}^{*}} \otimes 1+1 \otimes \operatorname{ad} e_{j}^{*}\right) \beta^{*}\left(e_{i}^{*}\right)
$$

where $\beta\left(e_{i}^{*}\right)=\sum_{k, l} c_{k l}^{i} e_{k}^{*} \otimes e_{l}^{*}$. Let both sides of the above equation act on $e_{m} \otimes e_{n}$ and after rearranging the terms suitably, we have

$$
(F 1)+(F 2)+(F 3)+(F 4)+(F 5)+(F 6)=0,
$$

where

$$
\begin{aligned}
& (F 1)=\sum_{t, l}\left(a_{t l}-a_{l t}\right)\left(c_{n t}^{j} c_{m l}^{i}-c_{n t}^{i} c_{m l}^{j}\right) ; \\
& (F 2)=\sum_{t, l}\left(-a_{l t} c_{t n}^{i} c_{m l}^{j}+a_{t l} c_{m t}^{j} c_{l n}^{i}-a_{t l} c_{m t}^{i} c_{l n}^{j}+a_{l t} c_{m l}^{i} c_{t n}^{j}\right) \\
& (F 3)=\sum_{t, l}\left(a_{t j}-a_{j t}\right)\left(c_{l t}^{i} c_{m n}^{l}-c_{n t}^{l} c_{m l}^{i}\right) ; \\
& (F 4)=\sum_{t, l} a_{j t}\left[c_{t l}^{i} c_{m n}^{l}-c_{t n}^{l} c_{m l}^{i}+\left(c_{m t}^{l}-c_{t m}^{l}\right) c_{l n}^{i}\right]
\end{aligned}
$$




$$
\begin{aligned}
& (F 5)=\sum_{t, l}\left(a_{t i}-a_{i t}\right)\left(c_{n t}^{l} c_{m l}^{j}-c_{l t}^{j} c_{m n}^{l}\right) ; \\
& (F 6)=\sum_{t, l} a_{i t}\left[-c_{t l}^{j} c_{m n}^{l}+c_{t n}^{l} c_{m l}^{j}-\left(c_{m t}^{l}-c_{t m}^{l}\right) c_{l n}^{j}\right] .
\end{aligned}
$$

$(F 1)$ is the coefficient of $e_{i} \otimes e_{j}$ in

$$
\left(-L_{e_{m}} \otimes L_{e_{n}}-L_{e_{n}} \otimes L_{e_{m}}\right) \sum_{t, l}\left(a_{t l}-a_{l t}\right) e_{t} \otimes e_{l}=\left(-L_{e_{m}} \otimes L_{e_{n}}-L_{e_{n}} \otimes L_{e_{m}}\right]\left(r_{12}-r_{21}\right)
$$

$(F 2)=0$ by interchanging the indices $t$ and $l$;

$(F 3)$ is the coefficient of $e_{i} \otimes e_{j}$ in

$$
\left(L_{e_{m} \cdot e_{n}} \otimes 1-L_{e_{m}} L_{e_{n}} \otimes 1\right) \sum_{t, l}\left(a_{t l}-a_{l t}\right) e_{t} \otimes e_{l}=\left[L_{e_{m} \cdot e_{n}} \otimes 1-L_{e_{m}} L_{e_{n}} \otimes 1\right]\left(r_{12}-r_{21}\right) ;
$$

$(F 4)=0$ since the term in the bracket is the coefficient of $e_{i}$ in $\left(e_{m}, e_{t}, e_{n}\right)-\left(e_{t}, e_{m}, e_{n}\right)=0$. $(F 5)$ is the coefficient of $e_{i} \otimes e_{j}$ in

$$
\left(1 \otimes L_{e_{m} \cdot e_{n}}-1 \otimes L_{e_{m}} L_{e_{n}}\right) \sum_{t, l}\left(a_{t l}-a_{l t}\right) e_{t} \otimes e_{l}=\left[1 \otimes L_{e_{m} \cdot e_{n}}-1 \otimes L_{e_{m}} L_{e_{n}}\right]\left(r_{12}-r_{21}\right) ;
$$

$F(6)=0$ since the term in the bracket is the coefficient of $e_{j}$ in $\left(e_{t}, e_{m}, e_{n}\right)-\left(e_{m}, e_{t}, e_{n}\right)=0$. Therefore we have

$$
\left[P\left(e_{m} \cdot e_{n}\right)-P\left(e_{m}\right) P\left(e_{n}\right)\right]\left(r_{12}-r_{21}\right)=0 .
$$

Hence the conclusion holds.

For any linear map $\alpha: A \rightarrow A \otimes A$, let $J_{\alpha}: A \rightarrow A \otimes A \otimes A$ be a linear map given by

$$
J_{\alpha}(x)=(\alpha \otimes \mathrm{id}) \alpha(x)-(\mathrm{id} \otimes \alpha) \alpha(x)-(\sigma \otimes \mathrm{id})(\alpha \otimes \mathrm{id}) \alpha(x)+(\sigma \otimes \mathrm{id})(\mathrm{id} \otimes \alpha) \alpha(x), \forall x \in A
$$

Lemma 5.2 Let $A$ be a vector space and $\alpha: A \otimes A \rightarrow A$ be a linear map. Then $\alpha^{*}$ : $A^{*} \otimes A^{*} \rightarrow A^{*}$ defines a left-symmetric algebra structure on $A^{*}$ if and only if $J_{\alpha}=0$.

Proof We denote the product in $A^{*}$ by "o". Then for any $a^{*}, b^{*} \in A^{*}$, we have

$$
a^{*} \circ b^{*}=\alpha^{*}\left(a^{*} \otimes b^{*}\right)
$$

Hence the associator satisfies (for any $a^{*}, b^{*}, c^{*} \in A^{*}$ )

$$
\begin{aligned}
\left(a^{*}, b^{*}, c^{*}\right) & =\left[\alpha^{*}\left(\alpha^{*} \otimes \mathrm{id}\right)-\alpha^{*}\left(\mathrm{id} \otimes \alpha^{*}\right)\right]\left(a^{*} \otimes b^{*} \otimes c^{*}\right) \\
\left(b^{*}, a^{*}, c^{*}\right) & =\left[\alpha^{*}\left(\alpha^{*} \otimes \mathrm{id}\right)-\alpha^{*}\left(\mathrm{id} \otimes \alpha^{*}\right)\right]\left(b^{*} \otimes a^{*} \otimes c^{*}\right) \\
& =\left[\alpha^{*}\left(\alpha^{*} \otimes \mathrm{id}\right)-\alpha^{*}\left(\mathrm{id} \otimes \alpha^{*}\right)\right](\sigma \otimes \mathrm{id})\left(a^{*} \otimes b^{*} \otimes c^{*}\right) .
\end{aligned}
$$


Therefore $\left(a^{*}, b^{*}, c^{*}\right)=\left(b^{*}, a^{*}, c^{*}\right)$ for any $a^{*}, b^{*}, c^{*} \in A^{*}$ if and only if $J_{\alpha}=0$.

Proposition 5.3 Let $(A, \cdot)$ be a left-symmetric algebra. Let $r=\sum_{i} a_{i} \otimes b_{i} \in A \otimes A$, where $a_{i}, b_{i} \in A$. Define $\alpha: A \rightarrow A \otimes A$ by equation (5.1). Then

$$
J_{\alpha}(x)=Q(x)[[r, r]]+\sum_{j}\left[P\left(x \cdot a_{j}\right)-P(x) P\left(a_{j}\right)\right]\left(r_{12}-r_{21}\right) \otimes b_{j}, \forall x \in A,
$$

where

$$
[[r, r]]=r_{13} \cdot r_{12}-r_{23} \cdot r_{21}+\left[r_{23}, r_{12}\right]-\left[r_{13}, r_{21}\right]-\left[r_{13}, r_{23}\right]
$$

and $Q(x)=L_{x} \otimes 1 \otimes 1+1 \otimes L_{x} \otimes 1+1 \otimes 1 \otimes \operatorname{ad} x, P(x)=L_{x} \otimes 1+1 \otimes L_{x}$ for any $x \in A$.

Before proving this result, let us explain the notations. Let $(A, \cdot)$ be a left-symmetric algebra and $r=\sum_{i} a_{i} \otimes b_{i}$, set

$r_{12}=\sum_{i} a_{i} \otimes b_{i} \otimes 1, r_{21}=\sum_{i} b_{i} \otimes a_{i} \otimes 1 ; r_{13}=\sum_{i} a_{i} \otimes 1 \otimes b_{i} ; r_{23}=\sum_{i} 1 \otimes a_{i} \otimes b_{i} \in U(\mathcal{G}(A))$,

where $U(\mathcal{G}(A))$ is the universal enveloping algebra of the sub-adjacent Lie algebra $\mathcal{G}(A)$. Set

$$
\begin{aligned}
& r_{13} \cdot r_{12}=\sum_{i, j} a_{i} \cdot a_{j} \otimes b_{j} \otimes b_{i} ; r_{23} \cdot r_{21}=\sum_{i, j} b_{j} \otimes a_{i} \cdot a_{j} \otimes b_{i} ; \\
& {\left[r_{23}, r_{12}\right]=r_{23} \cdot r_{12}-r_{12} \cdot r_{23}=\sum_{i j} a_{j} \otimes\left[a_{i}, b_{j}\right] \otimes b_{i} ;} \\
& {\left[r_{13}, r_{21}\right]=r_{13} \cdot r_{21}-r_{21} \cdot r_{13}=\sum_{i j}\left[a_{i}, b_{j}\right] \otimes a_{j} \otimes b_{i} ;} \\
& {\left[r_{13}, r_{23}\right]=r_{13} \cdot r_{23}-r_{23} \cdot r_{13}=\sum_{i j} a_{i} \otimes a_{j} \otimes\left[b_{i}, b_{j}\right] .}
\end{aligned}
$$

Proof Let $x \in A$. After rearranging the terms suitably, we divide $J_{\alpha}(x)$ into three parts:

$$
J_{\alpha}(x)=(F 1)+(F 2)+(F 3)
$$

where

$$
\begin{aligned}
(F 1)= & \sum_{i, j}\left\{\left(x \cdot a_{j}\right) \cdot a_{i} \otimes b_{i}-b_{i} \otimes\left(x \cdot a_{j}\right) \cdot a_{i}+b_{i} \cdot a_{j} \otimes x \cdot a_{i}-x \cdot a_{i} \otimes b_{i} \cdot a_{j}\right. \\
& \quad+a_{i} \otimes\left[x \cdot a_{j}, b_{i}-a_{i} \otimes\left[x, b_{i}\right] \cdot a_{j}+\left[x, b_{i}\right] \cdot a_{j} \otimes a_{i}-\left[x \cdot a_{j}, b_{i}\right] \otimes a_{i}\right\} \otimes b_{j} ; \\
(F 2)= & \sum_{i, j}\left\{a_{j} \cdot a_{i} \otimes b_{i}+a_{i} \otimes\left[a_{j}, b_{i}\right]-b_{i} \otimes a_{j} \cdot a_{i}-\left[a_{j}, b_{i}\right] \otimes a_{i}\right\} \otimes\left[x, b_{j}\right] \\
(F 3)= & \sum_{i, j}\left\{-x \cdot a_{i} \otimes a_{j} \otimes\left[b_{i}, b_{j}\right]-a_{i} \otimes a_{j} \otimes\left[\left[x, b_{i}\right], b_{j}\right]-a_{i} \otimes x \cdot a_{j} \otimes\left[b_{i}, b_{j}\right]\right. \\
& \left.\left.+a_{i} \otimes a_{j} \otimes\left[\left[x, b_{j}\right], b_{i}\right]\right]\right\} .
\end{aligned}
$$


On the other hand,

$$
\begin{gathered}
Q(x)\left(r_{13} \cdot r_{12}\right)=\sum_{i, j}\left\{x \cdot\left(a_{j} \cdot a_{i}\right) \otimes b_{i} \otimes b_{j}+a_{j} \cdot a_{i} \otimes x \cdot b_{i} \otimes b_{j}+a_{j} \cdot a_{i} \otimes b_{i} \otimes\left[x, b_{j}\right]\right\} ; \\
Q(x)\left(-r_{23} \cdot r_{21}\right)=\sum_{i, j}-\left\{x \cdot b_{i} \otimes a_{j} \cdot a_{i} \otimes b_{j}+b_{i} \otimes x \cdot\left(a_{j} \cdot a_{i}\right) \otimes b_{j}+b_{i} \otimes a_{j} \cdot a_{i} \otimes\left[x, b_{j}\right]\right\} ; \\
Q(x)\left(\left[r_{23}, r_{12}\right]\right)=\sum_{i, j}\left\{x \cdot a_{i} \otimes\left[a_{j}, b_{i}\right] \otimes b_{j}+a_{i} \otimes x \cdot\left[a_{j}, b_{i}\right] \otimes b_{j}+a_{i} \otimes\left[a_{j}, b_{i}\right] \otimes\left[x, b_{j}\right]\right\} ; \\
Q(x)\left(-\left[r_{13}, r_{21}\right]\right)=\sum_{i, j}-\left\{x \cdot\left[a_{j}, b_{i}\right] \otimes a_{i} \otimes b_{j}+\left[a_{j}, b_{i}\right] \otimes x \cdot a_{i} \otimes b_{j}+\left[a_{j}, b_{i}\right] \otimes a_{i} \otimes\left[x, b_{j}\right]\right\} ; \\
\begin{array}{c}
Q(x)\left(-\left[r_{13}, r_{23}\right]\right)= \\
\sum_{j}\left[P\left(x \cdot a_{j}\right)-P(x) P\left(a_{j}\right)\right]\left(r_{12}-r_{21}\right) \otimes b_{j}=\sum_{i, j}\left\{\left(x \cdot a_{j}\right) \cdot a_{i} \otimes b_{i}+a_{i} \otimes\left(x \cdot a_{j}\right) \cdot b_{i}\right. \\
-x \cdot a_{i} \otimes a_{j} \cdot b_{i}-a_{j} \cdot a_{i} \otimes x \cdot b_{i}-x \cdot\left(a_{j} \cdot a_{i}\right) \otimes b_{i}-a_{i} \otimes x \cdot\left(a_{j} \cdot b_{i}\right) \\
\left.-\left(x \cdot a_{j}\right) \cdot b_{i} \otimes a_{i}-b_{i} \otimes\left(x \cdot a_{j}\right) \cdot a_{i}+x \cdot b_{i} \otimes a_{j} \cdot a_{i}+a_{j} \cdot b_{i} \otimes x \cdot a_{i}\right]+a_{i} \otimes a_{j} \otimes\left[x, b_{i}\right. \\
\left.+x \cdot\left(a_{j} \cdot b_{i}\right) \otimes a_{i}+b_{i} \otimes x \cdot\left(a_{j} \cdot a_{i}\right)\right\} \otimes b_{j}
\end{array}
\end{gathered}
$$

After rearranging the terms suitably, the sum of the terms whose third component is $b_{j}$ in the right hand side of equation (5.4) is

$$
\left(F 1^{\prime}\right)=(F 1 a)+(F 1 b)+(F 1 c)+(F 1 d)+(F 1 e)+(F 1 f)+(F 1 g),
$$

where

$$
\begin{aligned}
(F 1 a) & =\sum_{i, j}\left\{x \cdot\left(a_{j} \cdot a_{i}\right)+\left(x \cdot a_{j}\right) \cdot a_{i}-x \cdot\left(a_{j} \cdot a_{i}\right)\right\} \otimes b_{i} \otimes b_{j}=\sum_{i, j}\left(x \cdot a_{j}\right) \cdot a_{i} \otimes b_{i} \otimes b_{j} ; \\
(F 1 b) & =\sum_{i, j} b_{i} \otimes\left\{-x \cdot\left(a_{j} \cdot a_{i}\right)-\left(x \cdot a_{j}\right) \cdot a_{i}+x \cdot\left(a_{j} \cdot a_{i}\right)\right\} \otimes b_{j}=\sum_{i, j}-b_{i} \otimes\left(x \cdot a_{j}\right) \cdot a_{i} \otimes b_{j} ; \\
(F 1 c) & =\sum_{i, j}\left\{-\left[a_{j}, b_{i}\right] \otimes x \cdot a_{i}+a_{j} \cdot b_{i} \otimes x \cdot a_{i}\right\} \otimes b_{j}=\sum_{i, j} b_{i} \cdot a_{j} \otimes x \cdot a_{i} \otimes b_{j} ; \\
(F 1 d) & =\sum_{i, j}\left\{x \cdot a_{i} \otimes\left[a_{j}, b_{i}\right]-x \cdot a_{i} \otimes a_{j} \cdot b_{i}\right\} \otimes b_{j}=\sum_{i, j}-x \cdot a_{i} \otimes b_{i} \cdot a_{j} \otimes b_{j} ; \\
(F 1 e) & =\sum_{i j}\left\{a_{i} \otimes x \cdot\left[a_{j}, b_{i}\right]+a_{i} \otimes\left(x \cdot a_{j}\right) \cdot b_{i}-a_{i} \otimes x \cdot\left(a_{j} \cdot b_{i}\right)\right\} \otimes b_{j} \\
& =\sum_{i, j} a_{i} \otimes\left\{-x \cdot\left(b_{i} \cdot a_{j}\right)+\left(x \cdot a_{j}\right) \cdot b_{i}\right\} \otimes b_{j}=\sum_{i, j} a_{i} \otimes\left\{\left[x \cdot a_{j}, b_{i}\right]-\left[x, b_{i}\right] \cdot a_{j}\right\} \otimes b_{j} ; \\
(F 1 f) & =\sum_{i, j}\left\{-x \cdot\left[a_{j}, b_{i}\right] \otimes a_{i}-\left(x \cdot a_{j}\right) \cdot b_{i} \otimes a_{i}+x \cdot\left(a_{j} \cdot b_{i}\right) \otimes a_{i}\right\} \otimes b_{j} \\
& =\sum_{i, j}\left\{x \cdot\left(b_{i} \cdot a_{j}\right)-\left(x \cdot a_{j}\right) \cdot b_{i}\right\} \otimes a_{i} \otimes b_{j}=\sum_{i, j}\left\{\left[x, b_{i}\right] \cdot a_{j}-\left[x \cdot a_{j}, b_{i}\right]\right\} \otimes a_{i} \otimes b_{j} ;
\end{aligned}
$$


$(F 1 g)=\sum_{i, j}\left\{a_{j} \cdot a_{i} \otimes x \cdot b_{i}-x \cdot b_{i} \otimes a_{j} \cdot a_{i}-a_{j} \cdot a_{i} \otimes x \cdot b_{i}+x \cdot b_{i} \otimes a_{j} \cdot a_{i}\right\} \otimes b_{j}=0$.

Therefore $\left(F 1^{\prime}\right)=(F 1)$. Obviously, the sum of the terms whose third component is $\left[x, b_{j}\right]$ in the right hand side of equation (5.4) is just (F2). Moreover, the sum of the other terms in the right hand side of equation (5.4) is $Q(x)\left(-\left[r_{13}, r_{23}\right]\right)$, which precisely equals to $(F 3)$ by Jacobi identity in the sub-adjacent Lie algebra $\mathcal{G}(A)$. Hence equation (5.4) holds.

With the discussion above together, we have the following result.

Theorem 5.4 Let $A$ be a left-symmetric algebra and $r \in A \otimes A$. Then the map $\alpha$ defined by equation (5.1) induces a left-symmetric algebra structure on $A^{*}$ such that $\left(A, A^{*}\right)$ is a leftsymmetric bialgebra if and only if the following two conditions are satisfied:

(a) $[P(x \cdot y)-P(x) P(y)]\left(r_{12}-r_{21}\right)=0$ for any $x, y \in A$;

(b) $Q(x)[[r, r]]=0$,

where $[[r, r]]$ is given by equation (5.5) and $Q(x)=L_{x} \otimes 1 \otimes 1+1 \otimes L_{x} \otimes 1+1 \otimes 1 \otimes \operatorname{ad} x$, $P(x)=L_{x} \otimes 1+1 \otimes L_{x}$ for any $x \in A$.

A direct application of Theorem 5.4 is given as follows (cf. Example 4.3).

Theorem 5.5 Let $\left(A, A^{*}, \alpha, \beta\right)$ be a left-symmetric bialgebra. Then there is a canonical left-symmetric bialgebra structure on $A \oplus A^{*}$ such that both the inclusions $i_{1}: A \rightarrow A \oplus A^{*}$ and $i_{2}: A^{*} \rightarrow A \oplus A^{*}$ into the two summands are homomorphisms of left-symmetric bialgebras.

Proof Let $r \in A \otimes A^{*} \subset\left(A \oplus A^{*}\right) \otimes\left(A \oplus A^{*}\right)$ correspond to the identity map $i d: A \rightarrow A$. Let $\left\{e_{1}, \cdots, e_{n}\right\}$ be a basis of $A$ and $\left\{e_{1}^{*}, \cdots, e_{n}^{*}\right\}$ be its dual basis. Then $r=\sum_{i} e_{i} \otimes e_{i}^{*}$. Suppose that the left-symmetric algebra structure "*” on $A \oplus A^{*}$ is given by $\mathcal{S D}(A)=A \bowtie_{\mathrm{ad}_{\circ}^{*},-R_{\circ}^{*}}^{\mathrm{a}{ }^{*},-R^{*}} A^{*}$. Then by Theorem 3.5, we have

$$
x * y=x \cdot y, a^{*} * b^{*}=a^{*} \circ b^{*}, x * a^{*}=\operatorname{ad}_{.}^{*}(x) a^{*}-R_{\circ}^{*}\left(a^{*}\right) x, a^{*} * x=\operatorname{ad}_{\circ}\left(a^{*}\right) x-R_{.}^{*}(x) a^{*},
$$

for any $x, y \in A, a^{*}, b^{*} \in A^{*}$. Next we prove that $r$ satisfies the two conditions in Theorem 5.4. If so, then

$$
\alpha_{\mathcal{S D}}(u)=\left(L_{u} \otimes 1+1 \otimes \operatorname{ad} u\right)(r), \quad \forall u \in \mathcal{S D}(A)
$$

can induce a left-symmetric bialgebra structure on $\mathcal{S D}(A)$.

For any $\lambda, \mu \in \mathcal{S D}(A)$, equation (5.2) is equivalent to

$$
\begin{aligned}
& \sum_{i}\left\{(\lambda * \mu) * e_{i} \otimes e_{i}^{*}-\lambda *\left(\mu * e_{i}\right) \otimes e_{i}^{*}-(\lambda * \mu) * e_{i}^{*} \otimes e_{i}+\lambda *\left(\mu * e_{i}^{*}\right) \otimes e_{i}\right. \\
& +e_{i} \otimes(\lambda * \mu) * e_{i}^{*}-e_{i} \otimes \lambda *\left(\mu * e_{i}^{*}\right)+e_{i}^{*} \otimes \lambda *\left(\mu * e_{i}\right)-e_{i}^{*} \otimes(\lambda * \mu) * e_{i} \\
& \left.-\lambda * e_{i} \otimes \mu * e_{i}^{*}+\mu * e_{i}^{*} \otimes \lambda * e_{i}+\lambda * e_{i}^{*} \otimes \mu * e_{i}-\mu * e_{i} \otimes \lambda * e_{i}^{*}\right\}=0
\end{aligned}
$$


We can prove the equation above in the following four cases: (I) $\lambda, \mu \in A$; (II) $\lambda, \mu \in A^{*}$; (III) $\lambda \in A, \mu \in A^{*}$ and (IV) $\lambda \in A^{*}, \mu \in A$. As an example, we give a detailed proof of the first case (the proof of other cases is similar). Let $\lambda=e_{k}, \mu=e_{l}$, then we have

$$
\begin{aligned}
\sum_{i} e_{i} \otimes\left(e_{k} * e_{l}\right) * e_{i}^{*} & =\sum_{i}-\left[e_{k} \cdot e_{l}, e_{i}\right] \otimes e_{i}^{*}+\sum_{i, m}\left\langle e_{m}^{*} \circ e_{i}^{*}, e_{k} \cdot e_{l}\right\rangle e_{i} \otimes e_{m} ; \\
\sum_{i}-\left(e_{k} * e_{l}\right) * e_{i}^{*} \otimes e_{i} & =\sum_{i} e_{i}^{*} \otimes\left[e_{k} \cdot e_{l}, e_{i}\right]-\sum_{i, m}\left\langle e_{m}^{*} \circ e_{i}^{*}, e_{k} \cdot e_{l}\right\rangle e_{m} \otimes e_{i} ; \\
\sum_{i}-e_{i} \otimes e_{k} *\left(e_{l} * e_{i}^{*}\right) & =\sum_{i}-\left[e_{l},\left[e_{k}, e_{i}\right]\right] \otimes e_{i}^{*}-\sum_{i, m}\left\langle e_{m}^{*} \circ e_{i}^{*}, e_{l}\right\rangle e_{i} \otimes e_{k} \cdot e_{m} \\
& +\sum_{i, m, n}\left\langle\left[e_{l}, e_{m}\right], e_{i}^{*}\right\rangle\left\langle e_{n}^{*} \circ e_{m}^{*}, e_{k}\right\rangle e_{i} \otimes e_{n} ; \\
\sum_{i} e_{k} *\left(e_{l} * e_{i}^{*}\right) \otimes e_{i} & =\sum_{i} e_{i}^{*} \otimes\left[e_{l},\left[e_{k}, e_{i}\right]\right]+\sum_{i, m}\left\langle e_{m}^{*} \circ e_{i}^{*}, e_{l}\right\rangle e_{k} \cdot e_{m} \otimes e_{i} \\
\sum_{i}-e_{k} * e_{i} \otimes e_{l} * e_{i}^{*} & \left.=\sum_{i, m, n}\left\langle\left[e_{l}, e_{m}\right], e_{i}^{*}\right\rangle\left\langle e_{n}^{*} \circ e_{m}^{*}, e_{k}\right\rangle e_{n} \otimes e_{i}, e_{i}\right] \otimes e_{i}^{*}-\sum_{i, m}\left\langle e_{m}^{*} \circ e_{i}^{*}, e_{l}\right\rangle e_{k} \cdot e_{i} \otimes e_{m} ; \\
\sum_{i} e_{l} * e_{i}^{*} \otimes e_{k} * e_{i} & =\sum_{i}-e_{i}^{*} \otimes e_{k} \cdot\left[e_{l}, e_{i}\right]+\sum_{i, m}\left\langle e_{m}^{*} \circ e_{i}^{*}, e_{l}\right\rangle e_{m} \otimes e_{k} \cdot e_{i} ; \\
\sum_{i}-e_{l} * e_{i} \otimes e_{k} * e_{i}^{*} & =\sum_{i} e_{l} \cdot\left[e_{k}, e_{i}\right] \otimes e_{i}^{*}-\sum_{i, m}\left\langle e_{m}^{*} \circ e_{i}^{*}, e_{k}\right\rangle e_{l} \cdot e_{i} \otimes e_{m} ; \\
\sum_{i} e_{k} * e_{i}^{*} \otimes e_{l} * e_{i} & =\sum_{i}-e_{i}^{*} \otimes e_{l} \cdot\left[e_{k}, e_{i}\right]+\sum_{i, m}\left\langle e_{m}^{*} \circ e_{i}^{*}, e_{k}\right\rangle e_{m} \otimes e_{l} \cdot e_{i} .
\end{aligned}
$$

The sum of the terms which are in $A \otimes A^{*}$ or $A^{*} \otimes A$ is zero since

$$
\begin{aligned}
& \left(e_{k} \cdot e_{l}\right) \cdot e_{i}-e_{k} \cdot\left(e_{l} \cdot e_{i}\right)-\left[e_{k} \cdot e_{l}, e_{i}\right]-\left[e_{l},\left[e_{k}, e_{i}\right]\right]+e_{k} \cdot\left[e_{l}, e_{i}\right]+e_{l} \cdot\left[e_{k}, e_{i}\right] \\
& =e_{i} \cdot\left(e_{k} \cdot e_{l}\right)-\left(e_{i} \cdot e_{k}\right) \cdot e_{l}-e_{k}\left(e_{i} \cdot e_{l}\right)+\left(e_{k} \cdot e_{i}\right) \cdot e_{l}=0 .
\end{aligned}
$$

The coefficient of $e_{i} \otimes e_{m}$ in the sum of the terms which are in $A \otimes A$ is

$$
\begin{aligned}
\left\langle e_{m}^{*} \circ e_{i}^{*}, e_{k} \cdot e_{l}\right\rangle-\left\langle e_{i}^{*} \circ e_{m}^{*}, e_{k} \cdot e_{l}\right\rangle & \\
& -\sum_{n}\left\{\left\langle e_{n}^{*} \circ e_{i}^{*}, e_{l}\right\rangle\left\langle e_{m}^{*}, e_{k} \cdot e_{n}\right\rangle+\left\langle\left[e_{l}, e_{n}\right], e_{i}^{*}\right\rangle\left\langle e_{m}^{*} \circ e_{n}^{*}, e_{k}\right\rangle\right. \\
& \left\langle e_{i}^{*}, e_{k} \cdot e_{n}\right\rangle\left\langle e_{n}^{*} \circ e_{m}^{*}, e_{l}\right\rangle-\left\langle e_{m}^{*},\left[e_{l}, e_{n}\right]\right\rangle\left\langle e_{i}^{*} \circ e_{n}^{*}, e_{k}\right\rangle \\
& -\left\langle e_{i}^{*}, e_{k} \cdot e_{n}\right\rangle\left\langle e_{m}^{*} \circ e_{n}^{*}, e_{l}\right\rangle+\left\langle e_{m}^{*}, e_{k} \cdot e_{n}\right\rangle\left\langle e_{i}^{*} \circ e_{n}^{*}, e_{l}\right\rangle \\
& \left.-\left\langle e_{i}^{*}, e_{l} \cdot e_{n}\right\rangle\left\langle e_{m}^{*} \circ e_{n}^{*}, e_{k}\right\rangle+\left\langle e_{m}^{*}, e_{l} \cdot e_{n}\right\rangle\left\langle e_{i}^{*} \circ e_{n}^{*}, e_{k}\right\rangle\right\} \\
= & \left\langle-L_{.}^{*}\left(e_{k}\right)\left[e_{m}^{*}, e_{i}^{*}\right], e_{l}\right\rangle+\left\langle L_{.}^{*}\left(e_{k}\right) e_{m}^{*} \circ e_{i}^{*}, e_{l}\right\rangle+\left\langle-L_{.}^{*}\left(L_{\circ}^{*}\left(e_{m}^{*}\right) e_{k}\right) e_{i}^{*}, e_{l}\right\rangle
\end{aligned}
$$




$$
\begin{aligned}
& -\left\langle L_{.}^{*}\left(e_{k}\right) e_{i}^{*} \circ e_{m}^{*}, e_{l}\right\rangle+\left\langle L_{.}^{*}\left(L_{\circ}^{*}\left(e_{i}^{*}\right) e_{k}\right) e_{m}^{*}, e_{l}\right\rangle \\
& +\left\langle e_{m}^{*} \circ L_{.}^{*}\left(e_{k}\right) e_{i}^{*}, e_{l}\right\rangle-\left\langle e_{i}^{*} \circ L_{.}^{*}\left(e_{k}\right) e_{m}^{*}, e_{l}\right\rangle=0
\end{aligned}
$$

since $\left(\mathcal{G}(A), \mathcal{G}\left(A^{*}\right), L_{.}^{*}, L_{\circ}^{*}\right)$ is a matched pair of Lie algebras. Hence $P\left(e_{k} \cdot e_{l}\right)-P\left(e_{k}\right) P\left(e_{l}\right)\left(r_{12}-\right.$ $\left.r_{21}\right)=0$. Furthermore,

$$
\begin{aligned}
{[[r, r]]=} & \sum_{i, j}\left\{e_{i} \cdot e_{j} \otimes e_{j}^{*} \otimes e_{i}^{*}+e_{j} \otimes\left[e_{i}, e_{j}^{*}\right] \otimes e_{i}^{*}-e_{j}^{*} \otimes e_{i} \cdot e_{j} \otimes e_{i}^{*}\right. \\
& \left.-\left[e_{i}, e_{j}^{*}\right] \otimes e_{j} \otimes e_{i}^{*}-e_{i} \otimes e_{j} \otimes\left[e_{i}^{*}, e_{j}^{*}\right]\right\}
\end{aligned}
$$

Note that

$$
\begin{aligned}
& \sum_{i, j}\left\{e_{i} \cdot e_{j} \otimes e_{j}^{*} \otimes e_{i}^{*}+e_{j} \otimes L_{.}^{*}\left(e_{i}\right) e_{j}^{*} \otimes e_{i}^{*}\right\}=0 ; \\
& \sum_{i, j}\left\{-e_{j} \otimes L_{\circ}^{*}\left(e_{j}^{*}\right) e_{i} \otimes e_{i}^{*}+L_{\circ}^{*}\left(e_{j}^{*}\right) e_{i} \otimes e_{j} \otimes e_{i}^{*}-e_{i} \otimes e_{j} \otimes\left[e_{i}^{*}, e_{j}^{*}\right]\right\}=0 ; \\
& \sum_{i, j}\left\{-L_{.}^{*}\left(e_{i}\right) e_{j}^{*} \otimes e_{j} \otimes e_{i}^{*}-e_{j}^{*} \otimes e_{i} \cdot e_{j} \otimes e_{i}^{*}\right\}=0 .
\end{aligned}
$$

Therefore $[[r, r]]=0$. Hence $\mathcal{S D}(A)$ is a left-symmetric bialgebra.

For $e_{i} \in A$, we have

$$
\begin{aligned}
\alpha_{S D}\left(e_{i}\right) & =\sum_{j}\left\{e_{i} \cdot e_{j} \otimes e_{j}^{*}+e_{j} \otimes\left[e_{i}, e_{j}^{*}\right]\right\} \\
& =\sum_{j, m}\left\{e_{i} \cdot e_{j} \otimes e_{j}^{*}-\left\langle e_{j}^{*}, e_{i} \cdot e_{m}\right\rangle e_{j} \otimes e_{m}^{*}+\left\langle e_{j}^{*} \circ e_{m}^{*}, e_{i}\right\rangle e_{j} \otimes e_{m}\right\} \\
& =\sum_{j, m}\left\langle e_{j}^{*} \circ e_{m}^{*}, e_{i}\right\rangle e_{j} \otimes e_{m} \\
& =\alpha_{A}\left(e_{i}\right)
\end{aligned}
$$

Therefore the inclusion $i_{1}: A \rightarrow A \oplus A^{*}$ is a homomorphism of left-symmetric bialgebras. Similarly, the inclusion $i_{2}: A^{*} \rightarrow A \oplus A^{*}$ is also a homomorphism of left-symmetric bialgebras since $\alpha_{S D}\left(e_{i}^{*}\right)=\alpha_{A^{*}}\left(e_{i}^{*}\right)$.

Definition 5.2 Let $\left(A, A^{*}\right)$ be a left-symmetric bialgebra. With the left-symmetric bialgebra structure given in Theorem 5.5, $A \oplus A^{*}$ is called a symplectic double of $A$. We denote it by $\mathcal{S D}(A)$.

Therefore, by Theorem 5.5, we have the following conclusion.

Proposition 5.6 Let $\left(A, A^{*}\right)$ be a left-symmetric bialgebra. Then the symplectic double $\mathcal{S D}(A)$ of $A$ is a left-symmetric bialgebra and its sub-adjacent Lie algebra is a parakähler Lie algebra with the symplectic form $\omega_{p}$ given by equation $(2.16)$. 
At the end of this section, we would like to point out that, unlike the symmetry of 1-cocycle of $\mathcal{G}(A)$ and $\mathcal{G}\left(A^{*}\right)$ appearing in the definition of a left-symmetric bialgebra $\left(A, A^{*}\right)$, it is not necessary that $\beta$ is also a 1 -coboundary of $\mathcal{G}\left(A^{*}\right)$ for a coboundary left-symmetric bialgebra $\left(A, A^{*}, \alpha, \beta\right)$, where $\alpha$ is given by equation (5.1).

\section{$6 \quad S$-equation and its properties}

The simplest way to satisfy the two conditions of Theorem 5.4 is to assume that $r$ is symmetric and $[[r, r]]=0$. Note that

$$
[[r, r]]=r_{21} \cdot r_{13}-r_{12} \cdot r_{23}-\left[r_{13}, r_{23}\right]+r_{13} \cdot\left(r_{12}-r_{21}\right)+r_{23} \cdot\left(r_{12}-r_{21}\right)
$$

Therefore, by Theorem 5.4, we have the following conclusion.

Proposition 6.1 Let $A$ be a left-symmetric algebra and $r \in A \otimes A$. Suppose $r$ is symmetric. Then the map $\alpha$ defined by equation (5.1) induces a left-symmetric algebra structure on $A^{*}$ such that $\left(A, A^{*}\right)$ is a left-symmetric bialgebra if

$$
[[r, r]]=-r_{12} \cdot r_{13}+r_{12} \cdot r_{23}+\left[r_{13}, r_{23}\right]=0
$$

Definition 6.1 Let $A$ be a left-symmetric algebra and $r \in A \otimes A$. Then equation (6.2) is called $S$-equation in $A$.

Remark 1 Let the products on $\{1,2,3\}$ correspond to the products of $A$ : for example, for any $i, j, k=1,2,3,(i \cdot j) \cdot k$ or $i \cdot(j \cdot k)$ corresponds to the (order) product $(x \cdot y) \cdot z$ or $x \cdot(y \cdot z)$ of $A$ respectively, and so on. For any symmetric $r \in A \otimes A$, set (for any $r_{i j}, i<j, i, j=1,2,3$ )

$$
r_{i j} \cdot r_{j l} \longleftrightarrow j \cdot(i \cdot l), \quad r_{i j} \cdot r_{i l} \longleftrightarrow i \cdot(j \cdot l), r_{i j} \cdot r_{l j} \longleftrightarrow(i \cdot l) \cdot j, r_{i j} \cdot r_{l i} \longleftrightarrow(j \cdot l) \cdot i .
$$

Then the $S$-equation (6.2) in a left-symmetric algebra corresponds to the "left-symmetry" of the products. It is similar to the relation between the classical Yang-Baxter equation in a Lie algebra $\mathcal{G}$ and the Jacobi identity of $\mathcal{G}([\mathrm{BD}],[\mathrm{D}],[\mathrm{Se}])$.

Remark 2 Let $\sigma_{123}, \sigma_{132}: A \otimes A \otimes A \rightarrow A \otimes A \otimes A$ be two linear maps satisfying

$$
\sigma_{123}(x \otimes y \otimes z)=z \otimes x \otimes y, \quad \sigma_{132}(x \otimes y \otimes z)=y \otimes z \otimes x, \quad \forall x, y, z \in A .
$$

Suppose that $r$ is symmetric. Then it is easy to know that under the action of $\sigma_{123}$ and $\sigma_{132}$ respectively, the $S$-equation (6.2) turns to be

$$
\left[r_{12}, r_{13}\right]-r_{23} \cdot\left(r_{12}-r_{13}\right)=0
$$




$$
\left[r_{12}, r_{23}\right]-r_{13} \cdot\left(r_{12}-r_{23}\right)=0
$$

respectively.

Let $A$ be a vector space. For any $r \in A \otimes A, r$ can be regarded as a map from $A^{*}$ to $A$ in the following way:

$$
\left\langle u^{*} \otimes v^{*}, r\right\rangle=\left\langle u^{*}, r\left(v^{*}\right)\right\rangle, \quad \forall u^{*}, v^{*} \in A^{*} .
$$

Proposition 6.2 Let $(A, \cdot)$ be a left-symmetric and $r \in A \otimes A$ be a symmetric solution of $S$-equation in $A$. Then the left-symmetric algebra and its sub-adjacent Lie algebra structure on the symplectic double $\mathcal{S D}(A)$ can be given from the products in $A$ as follows:

(a) $a^{*} * b^{*}=a^{*} \circ b^{*}=-R_{\text {. }}^{*}\left(r\left(b^{*}\right)\right) a^{*}+\operatorname{ad}^{*}\left(r\left(a^{*}\right)\right) b^{*}$, for any $a^{*}, b^{*} \in A^{*}$;

(b) $\left[a^{*}, b^{*}\right]=a^{*} \circ b^{*}-b^{*} \circ a^{*}=L^{*}\left(r\left(a^{*}\right)\right) b^{*}-L^{*}\left(r\left(b^{*}\right)\right) a^{*}$, for any $a^{*}, b^{*} \in A^{*}$;

(c) $x * a^{*}=x \cdot r\left(a^{*}\right)-r\left(\operatorname{ad}^{*}(x) a^{*}\right)+\operatorname{ad}^{*}(x) a^{*}$, for any $x \in A, a^{*} \in A^{*}$;

(d) $a^{*} * x=r\left(a^{*}\right) \cdot x+r\left(R^{*}(x) a^{*}\right)-R^{*}(x) a^{*}$, for any $x \in A, a^{*} \in A^{*}$;

(e) $\left[x, a^{*}\right]=\left[x, r\left(a^{*}\right)\right]-r\left(L_{.}^{*}(x) a^{*}\right)+L^{*}(x) a^{*}$, for any $x \in A, a^{*} \in A^{*}$.

Proof Let $\left\{e_{1}, \cdots, e_{n}\right\}$ be a basis of $A$ and $\left\{e_{1}^{*}, \cdots, e_{n}^{*}\right\}$ be its dual basis. Suppose that $e_{i} \cdot e_{j}=\sum_{i, j} c_{i j}^{k} e_{k}$ and $r=\sum_{i, j} a_{i j} e_{i} \otimes e_{j}$, where $a_{i j}=a_{j i}$. Then from the proof of Proposition 5.1 , we know that (for any $k, l$ )

$$
\begin{aligned}
e_{k}^{*} \circ e_{l}^{*} & =\sum_{i, t}\left\{a_{t l} c_{i t}^{k}+a_{k t}\left(c_{i t}^{l}-c_{t i}^{l}\right)\right\} e_{i}^{*}=\sum_{i, t}\left\{a_{t l}\left\langle e_{i} \cdot e_{t}, e_{k}^{*}\right\rangle+a_{k t}\left\langle\left[e_{i}, e_{t}\right], e_{l}^{*}\right\rangle\right\} e_{i}^{*} \\
& =\sum_{i}\left\{\left\langle e_{i} \cdot r\left(e_{l}^{*}\right), e_{k}^{*}\right\rangle+\left\langle\left[e_{i}, r\left(e_{k}^{*}\right)\right], e_{l}^{*}\right\rangle\right\} e_{i}^{*} \\
& =-R_{\cdot}^{*}\left(r\left(e_{l}^{*}\right)\right) e_{k}^{*}+\operatorname{ad}_{*}^{*}\left(r\left(e_{k}^{*}\right)\right) e_{l}^{*} .
\end{aligned}
$$

Hence equation (6.4) holds. Therefore, we have

$$
\begin{aligned}
-R_{\circ}^{*}\left(e_{k}^{*}\right) e_{l} & =\sum_{i}\left\langle-R_{\circ}^{*}\left(e_{k}^{*}\right) e_{l}, e_{i}^{*}\right\rangle e_{i}=\sum_{i}\left\langle e_{l}, e_{i}^{*} \circ e_{k}^{*}\right\rangle e_{i} \\
& =\sum_{i}\left\langle e_{l},-R_{.}^{*}\left(r\left(e_{k}^{*}\right)\right) e_{i}^{*}+\operatorname{ad}_{.}^{*}\left(r\left(e_{i}^{*}\right)\right) e_{k}^{*}\right\rangle e_{i} \\
& =e_{l} \cdot r\left(e_{k}^{*}\right)-\sum_{i}\left\langle\left[r\left(e_{i}^{*}\right), e_{l}\right], e_{k}^{*}\right\rangle e_{i} \\
& =e_{l} \cdot r\left(e_{k}^{*}\right)-r\left(\operatorname{ad}_{*}^{*}\left(e_{l}\right) e_{k}^{*}\right) .
\end{aligned}
$$

Since $e_{l} * e_{k}^{*}=\operatorname{ad}^{*}\left(e_{l}\right) e_{k}^{*}-R_{\circ}^{*}\left(e_{k}^{*}\right) e_{l}$, we get equation (6.6). Similarly, we can prove equation (6.7). Equations (6.5) and (6.8) then follow immediately. 
Definition 6.2 Let $(A, \cdot)$ be a left-symmetric algebra. A bilinear form $\mathcal{B}: A \otimes A \rightarrow \mathbf{F}$ is called a 2-cocycle of $A$ if

$$
\mathcal{B}(x \cdot y, z)-\mathcal{B}(x, y \cdot z)=\mathcal{B}(y \cdot x, z)-\mathcal{B}(y, x \cdot z), \forall x, y, z \in A
$$

In fact, the above definition is precisely the definition of a 2-cocycle of a left-symmetric algebra into the trivial bimodule $\mathbf{F}$ ([SW]). It is equivalent to the following central extension: there exists a left-symmetric algebra structure on $A \oplus \mathbf{F} c$ given by

$$
x * y=x \cdot y+\mathcal{B}(x, y) c, x * c=c * x=c * c=0, \quad \forall x, y \in A,
$$

if and only if $\mathcal{B}$ is a 2-cocycle of $A$. Furthermore, for any 2-cocycle $\mathcal{B}$ of a left-symmetric algebra $A$, it is easy to know that $([\mathrm{Ku} 2])$

$$
\omega(x, y)=\mathcal{B}(x, y)-\mathcal{B}(y, x), \quad \forall x, y \in A,
$$

is a 2-cocycle of the sub-adjacent Lie algebra $\mathcal{G}(A)$.

Theorem 6.3 Let $A$ be a left-symmetric algebra and $r \in A \otimes A$. Suppose that $r$ is symmetric and nondegenerate. Then $r$ is a solution of $S$-equation in $A$ if and only if the inverse of the isomorphism $A^{*} \rightarrow A$ induced by $r$, regarded as a bilinear form $\mathcal{B}$ on $A$, is a 2-cocycle of $A$. That is, $\mathcal{B}(x, y)=\left\langle r^{-1} x, y\right\rangle$ for any $x, y \in A$.

Proof Let $r=\sum_{i} a_{i} \otimes b_{i}$. Since $r$ is symmetric, we have $\sum_{i} a_{i} \otimes b_{i}=\sum_{i} b_{i} \otimes a_{i}$. Therefore $r\left(v^{*}\right)=\sum_{i}\left\langle v^{*}, a_{i}\right\rangle b_{i}=\sum_{i}\left\langle v^{*}, b_{i}\right\rangle a_{i}$ for any $v^{*} \in A^{*}$. Since $r$ is nondegenerate, for any $x, y, z \in A$, there exist $u^{*}, v^{*}, w^{*} \in A^{*}$ such that $x=r\left(u^{*}\right), y=r\left(v^{*}\right), z=r\left(w^{*}\right)$. Therefore

$$
\begin{aligned}
\mathcal{B}(x \cdot y, z) & =\left\langle r\left(u^{*}\right) \cdot r\left(v^{*}\right), w^{*}\right\rangle=\sum_{i, j}\left\langle u^{*}, b_{i}\right\rangle\left\langle v^{*}, b_{j}\right\rangle\left\langle w^{*}, a_{i} \cdot a_{j}\right\rangle \\
& =\left\langle u^{*} \otimes v^{*} \otimes w^{*}, r_{13} \cdot r_{23}\right\rangle ; \\
-\mathcal{B}(x, y \cdot z) & =-\left\langle u^{*}, r\left(v^{*}\right) \cdot r\left(w^{*}\right)\right\rangle=\sum_{i, j}-\left\langle v^{*}, b_{i}\right\rangle\left\langle w^{*}, b_{j}\right\rangle\left\langle u^{*}, a_{i} \cdot a_{j}\right\rangle \\
& =\left\langle u^{*} \otimes v^{*} \otimes w^{*},-r_{12} \cdot r_{13}\right\rangle ; \\
-\mathcal{B}(y \cdot x, z) & =-\left\langle r\left(v^{*}\right) \cdot r\left(u^{*}\right), w^{*}\right\rangle=-\sum_{i, j}\left\langle v^{*}, b_{i}\right\rangle\left\langle u^{*}, b_{j}\right\rangle\left\langle w^{*}, a_{i} \cdot a_{j}\right\rangle \\
& =\left\langle u^{*} \otimes v^{*} \otimes w^{*},-r_{23} \cdot r_{13}\right\rangle ; \\
\mathcal{B}(y, x \cdot z) & =\left\langle v^{*}, r\left(u^{*}\right) \cdot r\left(w^{*}\right)\right\rangle=\sum_{i, j}\left\langle u^{*}, b_{i}\right\rangle\left\langle w^{*}, b_{j}\right\rangle\left\langle v^{*}, a_{i} \cdot a_{j}\right\rangle \\
& =\left\langle u^{*} \otimes v^{*} \otimes w^{*}, r_{12} \cdot r_{23}\right\rangle .
\end{aligned}
$$


Therefore $\mathcal{B}$ is a (symmetric) 2-cocycle of $A$ if and only if $\left\langle u^{*} \otimes v^{*} \otimes w^{*},[[r, r]]\right\rangle=0$ for any $u^{*}, v^{*}, w^{*} \in A^{*}$, if and only if $[[r, r]]=0$.

Corollary 6.4 Let $(A, \cdot)$ be a left-symmetric and $r \in A \otimes A$ be a nondegenerate symmetric solution of $S$-equation in $A$. Suppose the left-symmetric algebra structure "०" on $A^{*}$ is induced by $r$ from equation (6.4). Then we have

$$
a^{*} \circ b^{*}=r^{-1}\left(r\left(a^{*}\right) \cdot r\left(b^{*}\right)\right), \quad \forall a^{*}, b^{*} \in A^{*}
$$

Therefore $r: A^{*} \rightarrow A$ is an isomorphism of left-symmetric algebras.

Proof For any $x, y \in A$, set $\mathcal{B}(x, y)=\left\langle r^{-1}(x), y\right\rangle$. Then $\mathcal{B}$ is a 2-cocycle of $A$. For any $a^{*}, b^{*} \in A^{*}$ and $x \in A$, from Proposition 6.2 and the above theorem, we know that

$$
\begin{aligned}
\left\langle a^{*} \circ b^{*}, x\right\rangle & =\left\langle x \cdot r\left(b^{*}\right), a^{*}\right\rangle-\left\langle\left[r\left(a^{*}\right), x\right], b^{*}\right\rangle \\
& =\mathcal{B}\left(x \cdot r\left(b^{*}\right), r\left(a^{*}\right)\right)-\mathcal{B}\left(\left[r\left(a^{*}\right), x\right], r\left(b^{*}\right)\right)=\mathcal{B}\left(x, r\left(a^{*}\right) \cdot r\left(b^{*}\right)\right) \\
& =\left\langle r^{-1}\left(r\left(a^{*}\right) \cdot r\left(b^{*}\right)\right), x\right\rangle .
\end{aligned}
$$

Hence equation (6.12) holds and therefore $r$ is an isomorphism of left-symmetric algebras.

Definition 6.3 A left-symmetric algebra $A$ over the real field $\mathbf{R}$ is called Hessian if there exists a symmetric and positive definite 2-cocycle $\mathcal{B}$ of $A$, that is, there exists a 2 -cocycle $\mathcal{B}$ of $A$ which is an inner product.

In geometry, a Hessian manifold $M$ is a flat affine manifold provided with a Hessian metric $g$, that is, $g$ is a Riemannian metric such that for any each point $p \in M$ there exists a $C^{\infty}$-function $\varphi$ defined on a neighborhood of $p$ such that $g_{i j}=\frac{\partial^{2} \varphi}{\partial x^{i} \partial x^{j}}$. A Hessian left-symmetric algebra corresponds to an affine Lie group $G$ with a $G$-invariant Hessian metric ([Sh]).

Hence, we have the following conclusion.

Proposition 6.5 Let $(A, \mathcal{B})$ be a Hessian left-symmetric algebra with the inner product $\mathcal{B}$. Then there exists a basis $\left\{e_{1}, \cdots, e_{n}\right\}$ such that $\mathcal{B}\left(e_{i}, e_{j}\right)=\delta_{i j}$. Suppose $e_{i} \cdot e_{j}=\sum_{k} c_{i j}^{k} e_{k}$. Then

$$
c_{i j}^{k}-c_{j i}^{k}+c_{i k}^{j}-c_{j k}^{i}=0, \quad \forall i, j, k .
$$

Under this basis, the corresponding symmetric solution of $S$-equation in $A$ is given by

$$
r=\sum_{i}^{n} e_{i} \otimes e_{i} .
$$


Moreover, let $\left\{e_{1}^{*}, \cdots, e_{n}^{*}\right\}$ be the dual basis of $\left\{e_{1}, \cdots, e_{n}\right\}$. Then the left-symmetric algebra and its sub-adjacent Lie algebra structures on the symplectic double $\mathcal{S D}(A)$ are given by

$$
\begin{gathered}
e_{i}^{*} * e_{j}^{*}=r\left(e_{i} \cdot e_{j}\right)=\sum_{k}^{n} c_{i j}^{k} e_{k}^{*} ;\left[e_{i}^{*}, e_{j}^{*}\right]=r\left(\left[e_{i}, e_{j}\right]\right)=\sum_{k}^{n}\left(c_{i j}^{k}-c_{j i}^{k}\right) e_{k}^{*} ; \\
e_{i} * e_{j}^{*}=\sum_{k}^{n}\left[c_{k j}^{i} e_{k}+\left(c_{k i}^{j}-c_{i k}^{j}\right) e_{k}^{*}\right] ; e_{j}^{*} * e_{i}=\sum_{k}^{n}\left[\left(c_{j i}^{k}-c_{i j}^{k}\right) e_{k}+c_{k i}^{j} e_{k}^{*}\right] ;\left[e_{i}, e_{j}^{*}\right]=\sum_{k}^{n}\left(c_{j k}^{i} e_{k}-c_{i k}^{j} e_{k}^{*}\right) .
\end{gathered}
$$

On the other hand, if equation (6.14) is a symmetric solution of $S$-equation in a left-symmetric algebra $A$, then the structure constants $\left\{c_{i j}^{k}\right\}$ associated to the basis $\left\{e_{1}, \cdots, e_{n}\right\}$ satisfy equation (6.13).

Example 6.1 For an arbitrary nonassociative algebra $(A, \cdot)$, there is an "invariant" bilinear form $\mathcal{B}$ defined as a trace form $([\mathrm{Sc}])$ :

$$
\mathcal{B}(x \cdot y, z)=\mathcal{B}(x, y \cdot z) \text {, or equivalently, } \mathcal{B}\left(L_{x}(y), z\right)=\mathcal{B}\left(x, R_{y}(z)\right), \forall x, y, z \in A \text {. }
$$

Obviously, for a left-symmetric algebra $A$, a trace form is a 2-cocycle of $A$. Hence a nondegenerate symmetric trace form of a left-symmetric algebra $A$ can give a symmetric solution of $S$-equation in $A$. For example, $\mathbf{F}$ can be regarded as a 1-dimensional associative algebra with a basis $\{e\}$ satisfying $e \cdot e=e$. Then $r=e \otimes e$ as a solution of $S$-equation in $\mathbf{F}$ corresponds to the trace form $\mathcal{B}(e, e)=1$. Moreover, a left-symmetric algebra with a nondegenerate symmetric trace form must be associative and hence it is a Frobenius algebra ([Bo2]).

Example 6.2 Let $(A, \cdot)$ be a left-symmetric algebra. There is another "invariant" bilinear form $\mathcal{B}$ in the following sense $([\mathrm{BM} 4])$ :

$$
\mathcal{B}(x \cdot y, z)=-\mathcal{B}(y, x \cdot z), \quad \text { or equivalently, } \mathcal{B}\left(L_{x}(y), z\right)=-\mathcal{B}\left(y, L_{x}(z)\right), \forall x, y, z \in A
$$

The existence of such a nondegenerate bilinear form on $A$ is equivalent to the fact that $L$ is isomorphic to $L^{*}$ as representations of $\mathcal{G}(A)$. Obviously, such a nondegenerate symmetric bilinear form on a left-symmetric algebra $A$ is also a 2-cocycle of $A$. In fact, we have constructed some examples of parakähler Lie algebras from these bilinear forms in [Bai2]. Here, we explain why such a bilinear form appears in our study. As it was pointed in [BM4], such bilinear forms are degenerate on most of left-symmetric algebras.

Next we turn to the general symmetric solutions of $S$-equation. 
Theorem 6.6 Let $(A, \cdot)$ be a left-symmetric algebra and $r \in A \otimes A$ be symmetric. Then $r$ is a solution of $S$-equation in $A$ if and only if $r$ satisfies

$$
\left[r\left(a^{*}\right), r\left(b^{*}\right)\right]=r\left(L_{.}^{*}\left(r\left(a^{*}\right)\right) b^{*}-L_{.}^{*}\left(r\left(b^{*}\right)\right) a^{*}\right), \quad \forall a^{*}, b^{*} \in A^{*}
$$

Proof Let $\left\{e_{1}, \cdots, e_{n}\right\}$ be a basis of $A$ and $\left\{e_{1}^{*}, \cdots, e_{n}^{*}\right\}$ be its dual basis. Suppose that $e_{i} \cdot e_{j}=\sum_{k} c_{i j}^{k} e_{k}$ and $r=\sum_{i, j} a_{i j} e_{i} \otimes e_{j}, a_{i j}=a_{j i}$. Hence $r\left(e_{i}^{*}\right)=\sum_{k} a_{i k} e_{k}$. Then $r$ is a solution of $S$-equation in $A$ if and only if (for any $i, j, k$ )

$$
\sum_{t, l}\left\{-c_{t l}^{i} a_{t j} a_{l k}+c_{t l}^{j} a_{i t} a_{l k}+\left(c_{t l}^{k}-c_{l t}^{k}\right) a_{i t} a_{l j}\right\}=0
$$

The left-hand side of the above equation is precisely the coefficient of $e_{k}$ in

$$
\left[r\left(e_{i}^{*}\right), r\left(e_{j}^{*}\right)\right]-r\left(L_{.}^{*}\left(r\left(e_{i}^{*}\right)\right) e_{j}^{*}-L_{.}^{*}\left(r\left(e_{j}^{*}\right)\right) e_{i}^{*}\right)
$$

Therefore the conclusion follows.

Definition 6.4 ([Ku3]) Let $\mathcal{G}$ be a Lie algebra and $\rho: \mathcal{G} \rightarrow g l(V)$ be its representation. A linear map $T: V \rightarrow \mathcal{G}$ is called an $\mathcal{O}$-operator associated to $\rho$ if $T$ satisfies

$$
[T(u), T(v)]=T(\rho(T(u)) v-\rho(T(v)) u), \forall u, v \in V .
$$

Obviously, for a left-symmetric algebra $A$, the identity map $i d$ is an $\mathcal{O}$-operator associated to the regular representation of the sub-adjacent Lie algebra $\mathcal{G}(A)$. On the other hand,

Lemma 6.7 ([Bai3]) Let $\mathcal{G}$ be a Lie algebra and $\rho: \mathcal{G} \rightarrow g l(V)$ be its representation. Let $T: V \rightarrow \mathcal{G}$ be an $\mathcal{O}$-operator associated to $\rho$. Then the product

$$
u \circ v=\rho(T(u)) v, \quad \forall u, v \in V
$$

defines a left-symmetric algebra structure on $V$. Therefore $V$ is a Lie algebra as the subadjacent Lie algebra of this left-symmetric algebra and $T$ is a homomorphism of Lie algebras. Furthermore, $T(V)=\{T(v) \mid v \in V\} \subset \mathcal{G}$ is a Lie subalgebra of $\mathcal{G}$ and there is an induced left-symmetric algebra structure on $T(V)$ given by

$$
T(u) \cdot T(v)=T(u \circ v)=T(\rho(T(u)) v), \quad \forall u, v \in V .
$$

Moreover, its sub-adjacent Lie algebra structure is just the Lie subalgebra structure of $\mathcal{G}$ and $T$ is a homomorphism of left-symmetric algebras.

Therefore we have the following result. 
Corollary 6.8 Let $(A, \cdot)$ be a left-symmetric algebra. Let $r \in A \otimes A$ be a symmetric solution of $S$-equation in $A$. Then $r$ is an $\mathcal{O}$-operator associated to $L^{*}$. Therefore there is a left-symmetric algebra structure on $A^{*}$ given by

$$
a^{*} o^{\prime} b^{*}=L^{*}\left(r\left(a^{*}\right)\right) b^{*}, \forall a^{*}, b^{*} \in A^{*} .
$$

It has the same sub-adjacent Lie algebra of the left-symmetric algebra in $A^{*}$ given by equation (6.4), which is induced by $r$ in the sense of coboundary left-symmetric bialgebras. If $r$ is nondegenerate, then there is a new compatible left-symmetric algebra structure on $\mathcal{G}(A)$ given by

$$
x \cdot^{\prime} y=r\left(L^{*}(x) r^{-1} y\right), \quad \forall x, y \in A,
$$

which is just the left-symmetric algebra structure given by

$$
\mathcal{B}\left(x \cdot^{\prime} y, z\right)=-\mathcal{B}(y, x \cdot z), \forall x, y, z \in A,
$$

where $\mathcal{B}$ is the symmetric nondegenerate 2 -cocycle of $A$ induced by $r^{-1}$, that is, for any $x, y \in A$, $\mathcal{B}(x, y)=\left\langle r^{-1}(x), y\right\rangle$.

Remark With the above conditions and the left-symmetric algebra structure on $A^{*}$ given by equation (6.22), $\left(A, A^{*}\right)$ is a left-symmetric bialgebra if and only if the following two conditions hold: (for any $x, y \in A$ and $a^{*}, b^{*} \in A^{*}$ )

$$
\begin{gathered}
L^{*}\left[x \cdot r\left(a^{*}\right)-r\left(L_{.}^{*}(x) a^{*}\right)\right] b^{*}=L^{*}\left[x \cdot r\left(b^{*}\right)-r\left(L_{.}^{*}(x) b^{*}\right)\right] a^{*} ; \\
{\left[x \cdot r\left(a^{*}\right)-r\left(L^{*}(x) a^{*}\right)\right] \cdot y=\left[y \cdot r\left(a^{*}\right)-r\left(L^{*}(y) a^{*}\right)\right] \cdot x .}
\end{gathered}
$$

Theorem 6.9 Let $\mathcal{G}$ be a Lie algebra. Let $\rho: \mathcal{G} \rightarrow g l(V)$ be a representation of $\mathcal{G}$ and $\rho^{*}: \mathcal{G} \rightarrow g l\left(V^{*}\right)$ be its dual representation. Suppose that $T: V \rightarrow \mathcal{G}$ is an $\mathcal{O}$-operator associated to $\rho$. Then

$$
r=T+T^{21}
$$

is a symmetric solution of $S$-equation in $T(V) \ltimes_{\rho^{*}, 0} V^{*}$, where $T(V) \subset \mathcal{G}$ is a left-symmetric algebra given by equation $(6.21)$ and $\left(\rho^{*}, 0\right)$ is its bimodule since its sub-adjacent Lie algebra $\mathcal{G}(T(V))$ is a Lie subalgebra of $\mathcal{G}$, and $T$ can be identified as an element in $T(V) \otimes V^{*} \subset$ $\left(T(V) \ltimes_{\rho^{*}, 0} V^{*}\right) \otimes\left(T(V) \ltimes_{\rho^{*}, 0} V^{*}\right)$.

Proof Let $\left\{e_{1}, \cdots, e_{n}\right\}$ be a basis of $\mathcal{G}$. Let $\left\{v_{1}, \cdots, v_{m}\right\}$ be a basis of $V$ and $\left\{v_{1}^{*}, \cdots, v_{m}^{*}\right\}$ be its dual basis. Set $T\left(v_{i}\right)=\sum_{k=1}^{n} a_{i k} e_{k}, i=1, \cdots, m$. Then, we have

$$
T=\sum_{i=1}^{m} T\left(v_{i}\right) \otimes v_{i}^{*}=\sum_{i=1}^{m} \sum_{k=1}^{n} a_{i k} e_{k} \otimes v_{i}^{*} \in T(V) \otimes V^{*} \subset\left(T(V) \ltimes_{\rho^{*}, 0} V^{*}\right) \otimes\left(T(V) \ltimes_{\rho^{*}} V^{*}\right) .
$$


Therefore we have

$$
\begin{aligned}
-r_{12} \cdot r_{13}= & -\sum_{i, j=1}^{m}\left\{T\left(v_{i}\right) \cdot T\left(v_{j}\right) \otimes v_{i}^{*} \otimes v_{j}^{*}+v_{i}^{*} \otimes v_{j}^{*} \otimes T\left(\rho\left(T\left(v_{j}\right)\right) v_{i}\right)\right\} \\
r_{12} \cdot r_{23}= & \sum_{i, j=1}^{m}\left\{v_{i}^{*} \otimes T\left(v_{i}\right) \cdot T\left(v_{j}\right) \otimes v_{j}^{*}-v_{i}^{*} \otimes v_{j}^{*} \otimes T\left(\rho\left(T\left(v_{i}\right)\right) v_{j}\right)\right\} \\
{\left[r_{13}, r_{23}\right]=} & \sum_{i, j=1}^{m}\left\{-v_{i}^{*} \otimes T\left(\rho\left(T\left(v_{i}\right)\right) v_{j}\right) \otimes v_{j}^{*}+T\left(\rho\left(T\left(v_{j}\right)\right) v_{i}\right) \otimes v_{j}^{*} \otimes v_{i}^{*}\right. \\
& \left.+v_{i}^{*} \otimes v_{j}^{*} \otimes\left[T\left(v_{i}\right), T\left(v_{j}\right)\right]\right\} .
\end{aligned}
$$

Since $T$ is an $\mathcal{O}$-operator associated to $\rho$ and $T(u) \cdot T(v)=T(\rho(T(u)) v))$ for any $u, v \in V$, we know that $r$ is a symmetric solution of $S$-equation in $T(V) \ltimes_{\rho^{*}, 0} V^{*}$.

Remark The above theorem also provides a method to construct the symmetric solutions of $S$-equation (hence parakähler Lie algebras). We would like to point out that this method starts from Lie algebras and only involves the representation theory of Lie algebras (to find the $\mathcal{O}$-operators), which avoids involving the nonassociativity of left-symmetric algebras.

Corollary 6.10 Let $(A, \cdot)$ be a left-symmetric algebra. Then

$$
r=\sum_{i=1}^{n}\left(e_{i} \otimes e_{i}^{*}+e_{i}^{*} \otimes e_{i}\right)
$$

is a symmetric solution of $S$-equation in $A \ltimes_{L^{*}, 0} A^{*}$, where $\left\{e_{1}, \cdots, e_{n}\right\}$ is a basis of $A$ and $\left\{e_{1}^{*}, \cdots, e_{n}^{*}\right\}$ is its dual basis. Moreover, $r$ is nondegenerate and the induced 2-cocycle $\mathcal{B}$ of $A \ltimes_{L^{*}, 0} A^{*}$ is given by

$$
\mathcal{B}\left(x+a^{*}, y+b^{*}\right)=\left\langle r^{-1}\left(x+a^{*}\right), y+b^{*}\right\rangle=\left\langle x, b^{*}\right\rangle+\left\langle a^{*}, y\right\rangle, \quad \forall x, y \in A, a^{*}, b^{*} \in A^{*}
$$

Proof Let $V=A, \rho=L$ and $T=i d$ in Theorem 6.9. Then the conclusion follows immediately.

Remark Comparing with Theorem 5.5, we know that (the non-symmetric) $T=\sum_{i=1}^{n} e_{i} \otimes e_{i}^{*}$ induces a left-symmetric bialgebra structure on $A \ltimes_{\mathrm{ad}^{*},-R^{*}} A^{*}$, whereas the above (symmetric) $r=T+T^{21}$ induces a left-symmetric bialgebra structure on $A \ltimes_{L^{*}, 0} A^{*}$.

\section{Comparison between Lie bialgebras and left-symmetric bial- gebras}

In this section, we first recall some facts on Lie bialgebras from Chapters 1-2 in [CP] by Chari and Pressley. In fact, a Lie bialgebra is the Lie algebra $\mathcal{G}$ of a Poisson-Lie group $G$ equipped 
with additional structures induced from the Poisson structure on $G$ and a Poisson-Lie group is a Lie group with a Poisson structure compatible with the group operation in a certain sense. Poisson-Lie groups play an important role in symplectic geometry and quantum group theory $([\mathrm{D}],[\mathrm{KM}],[\mathrm{LW}])$.

Definition 7.1 Let $\mathcal{G}$ be a Lie algebra. A Lie bialgebra structure on $\mathcal{G}$ is a skew-symmetric linear map $\delta_{\mathcal{G}}: \mathcal{G} \rightarrow \mathcal{G} \otimes \mathcal{G}$ such that $\delta_{\mathcal{G}}^{*}: \mathcal{G}^{*} \otimes \mathcal{G}^{*} \rightarrow \mathcal{G}^{*}$ is a Lie bracket on $\mathcal{G}^{*}$ and $\delta$ is a 1 -cocycle of $\mathcal{G}$ associated to ad $\otimes 1+1 \otimes$ ad with values in $\mathcal{G} \otimes \mathcal{G}$. We denote it by $\left(\mathcal{G}, \mathcal{G}^{*}\right)$ or $\left(\mathcal{G}, \delta_{\mathcal{G}}\right)$.

Let $\mathcal{G}$ be a Lie algebra. A bilinear form $B($, ) on $\mathcal{G}$ is called invariant if

$$
B([x, y], z)=B(x,[y, z]), \quad \forall x, y, z \in \mathcal{G} .
$$

Definition 7.2 A Manin triple is a triple of Lie algebras $\left(\mathcal{P}, \mathcal{P}_{+}, \mathcal{P}_{-}\right)$together with a non-degenerate symmetric invariant bilinear form $B($,$) on \mathcal{P}$ such that

(1) $\mathcal{P}_{+}$and $\mathcal{P}_{-}$are Lie subalgebras of $\mathcal{P}$;

(2) $\mathcal{P}=\mathcal{P}_{+} \oplus \mathcal{P}_{-}$as vector spaces;

(3) $B\left(\mathcal{P}_{+}, \mathcal{P}_{+}\right)=B\left(\mathcal{P}_{-}, \mathcal{P}_{-}\right)=0$.

Two Manin triple $\left(\mathcal{P}_{1}, \mathcal{P}_{1,+}, \mathcal{P}_{1,-}\right)$ and $\left(\mathcal{P}_{2}, \mathcal{P}_{2,+}, \mathcal{P}_{2,-}\right)$ with the bilinear forms $B_{1}($,$) and B($, respectively are isomorphic if there exists an isomorphism of Lie algebras $\varphi: \mathcal{P}_{1} \rightarrow \mathcal{P}_{2}$ such that

$$
\varphi\left(\mathcal{P}_{1,+}\right)=\mathcal{P}_{2,+}, \quad \varphi\left(\mathcal{P}_{1,-}\right)=\mathcal{P}_{2,-}, B_{1}(x, y)=B_{2}(\varphi(x), \varphi(y)), \quad \forall x, y \in \mathcal{P}_{1}
$$

In particular, if there is a Lie algebra structure $\mathcal{G} \bowtie \mathcal{G}^{*}$ on $\mathcal{G} \oplus \mathcal{G}^{*}$ such that $\mathcal{G}$ and $\mathcal{G}^{*}$ are Lie subalgebras and the natural symmetric bilinear form (also see equation (6.29))

$$
\left(x+a^{*}, y+b^{*}\right)=\left\langle a^{*}, y\right\rangle+\left\langle x, b^{*}\right\rangle, \quad \forall x, y \in \mathcal{G}, a^{*}, b^{*} \in \mathcal{G}^{*},
$$

is invariant, then $\left(\mathcal{G} \bowtie \mathcal{G}^{*}, \mathcal{G}, \mathcal{G}^{*}\right)$ is a (standard) Manin triple. It is known that every Manin triple is isomorphic to such a standard Manin triple.

Theorem 7.1 Let $\left(\mathcal{G},[,]_{\mathcal{G}}\right)$ be a Lie algebra and $\left(\mathcal{G}^{*},[,]_{\mathcal{G}^{*}}\right)$ be a Lie algebra structure on its dual space $\mathcal{G}^{*}$. Then the following conditions are equivalent:

(1) $\left(\mathcal{G} \bowtie \mathcal{G}^{*}, \mathcal{G}, \mathcal{G}^{*}\right)$ is a standard Manin triple with the bilinear form (7.3);

(2) $\left(\mathcal{G}, \mathcal{G}^{*}, \operatorname{ad}_{\mathcal{G}}^{*}, \operatorname{ad}_{\mathcal{G}^{*}}^{*}\right)$ is a matched pair of Lie algebras;

$(3)\left(\mathcal{G}, \mathcal{G}^{*}\right)$ is a Lie bialgebra.

Proposition 7.2 Let $\left(\mathcal{G}, \mathcal{G}^{*}\right)$ be a Lie bialgebra. Then there is a canonical Lie bialgebra structure on $\mathcal{G} \oplus \mathcal{G}^{*}$ such that the inclusions $i_{1}: \mathcal{G} \rightarrow \mathcal{G} \oplus \mathcal{G}^{*}$ and $i_{2}: \mathcal{G}^{*} \rightarrow \mathcal{G} \oplus \mathcal{G}^{*}$ into the two 
summands are homomorphisms of Lie bialgebras. Such a structure is called a classical (Drinfeld) double of $\mathcal{G}$.

Definition 7.3 A Lie bialgebra $(\mathcal{G}, \delta)$ is called coboundary if there exists a $r \in \mathcal{G} \otimes \mathcal{G}$ such that

$$
\delta(x)=[x \otimes 1+1 \otimes x, r], \quad \forall x \in \mathcal{G} .
$$

Theorem 7.3 Let $\mathcal{G}$ be a Lie algebra and $r \in \mathcal{G} \otimes \mathcal{G}$. Then the map $\delta: \mathcal{G} \rightarrow \mathcal{G} \otimes \mathcal{G}$ defined by equation (7.4) induces a Lie bialgebra structure on $\mathcal{G}$ if and only if the following two conditions are satisfied:

(a) $\left[x \otimes 1+1 \otimes x, r_{12}+r_{21}\right]=0$ for any $x \in \mathcal{G}$;

(b) $\left[x \otimes 1 \otimes 1+1 \otimes x \otimes 1+1 \otimes 1 \otimes x, \quad\left[r_{12}, r_{13}\right]+\left[r_{12}, r_{23}\right]+\left[r_{13}, r_{23}\right]\right]=0$ for any $x \in \mathcal{G}$, where the notations $r_{12}, r_{21}, r_{13}, r_{23}$ are given as the notations after Proposition 5.3 for a Lie algebra.

Corollary 7.4 Let $\mathcal{G}$ be a Lie algebra and $r \in \mathcal{G} \otimes \mathcal{G}$. If $r$ is skew-symmetric and $r$ satisfies

$$
\left[r_{12}, r_{13}\right]+\left[r_{12}, r_{23}\right]+\left[r_{13}, r_{23}\right]=0
$$

then the map $\delta: \mathcal{G} \rightarrow \mathcal{G} \otimes \mathcal{G}$ defined by equation (7.4) induces a Lie bialgebra structure on $\mathcal{G}$.

Definition 7.4 Let $\mathcal{G}$ be a Lie algebra and $r \in \mathcal{G} \otimes \mathcal{G}$. Equation (7.5) is called classical Yang-Baxter equation $(\mathrm{CYBE})$ in $\mathcal{G}$.

The facts above are from Chapters 1-2 in $[\mathrm{CP}]$. Next we recall some results from the literature.

Proposition 7.5 Let $\mathcal{G}$ be a Lie algebra and $r \in \mathcal{G} \otimes \mathcal{G}$.

(1) [D] Suppose $r$ is skew-symmetric and nondegenerate. Then $r$ is a solution of CYBE in $\mathcal{G}$ if and only if the isomorphism $\mathcal{G}^{*} \rightarrow \mathcal{G}$ induced by $r$, regarded as a bilinear form on $\mathcal{G}$ is a 2 -cocycle of $\mathcal{G}$. Therefore under this situation, $\mathcal{G}$ is a symplectic Lie algebra.

(2) $([\mathrm{Ku} 3])$ Suppose $r$ is skew-symmetric. Then $r$ is a solution of CYBE in $\mathcal{G}$ if and only if $r$ is an $\mathcal{O}$-operator associated to $\mathrm{ad}^{*}$, that is, $r$ satisfies

$$
\left[r\left(a^{*}\right), r\left(b^{*}\right)\right]=r\left(\operatorname{ad}^{*}\left(r\left(a^{*}\right)\right) b^{*}-\operatorname{ad}^{*}\left(r\left(b^{*}\right)\right) a^{*}\right), \quad \forall a^{*}, b^{*} \in \mathcal{G}^{*} .
$$

Remark Let $\mathcal{G}$ be a Lie algebra equipped with a nondegenerate symmetric invariant bilinear form. Let $r \in \mathcal{G} \otimes \mathcal{G}$. Then $r$ can be identified as a linear map from $\mathcal{G}$ to $\mathcal{G}$. If $r$ is skew-symmetric, then $r$ is a solution of CYBE in $\mathcal{G}$ if and only if $r$ satisfies equation (2.7), that is, the operator form of CYBE. 
Proposition 7.6 ([Bai3]) $\quad$ Let $\mathcal{G}$ be a Lie algebra and $\rho: \mathcal{G} \rightarrow g l(V)$ be a representation of $\mathcal{G}$. Let $\rho^{*}: \mathcal{G} \rightarrow g l\left(V^{*}\right)$ be the dual representation of $\rho$ and $T: V \rightarrow \mathcal{G}$ be a linear map. Then

$$
r=T-T^{21}
$$

is a skew-symmetric solution of CYBE in $\mathcal{G} \ltimes_{\rho^{*}} V^{*}$ if and only if $T$ is an $\mathcal{O}$-operator associated to $\rho$.

Therefore, roughly speaking, a symmetric solution of the $S$-equation corresponds to the symmetric part of an $\mathcal{O}$-operator, whereas a skew-symmetric solution of the classical YangBaxter equation corresponds to the skew-symmetric part of an $\mathcal{O}$-operator.

Proposition $7.7([\mathrm{Bai} 3]) \quad$ Let $(A, \cdot)$ be a left-symmetric algebra. Then

$$
r=\sum_{i=1}^{n}\left(e_{i} \otimes e_{i}^{*}-e_{i}^{*} \otimes e_{i}\right)
$$

is a skew-symmetric solution of CYBE in $\mathcal{G}(A) \ltimes_{L^{*}} \mathcal{G}(A)^{*}$, where $\left\{e_{1}, \cdots, e_{n}\right\}$ is a basis of $A$ and $\left\{e_{1}^{*}, \cdots, e_{n}^{*}\right\}$ is its dual basis. Moreover, $r$ is nondegenerate and the induced 2-cocycle $\mathcal{B}$ of $\mathcal{G}(A) \ltimes_{L^{*}} \mathcal{G}(A)^{*}$ given by

$$
\mathcal{B}\left(x+a^{*}, y+b^{*}\right)=\left\langle r^{-1}\left(x+a^{*}\right), y+b^{*}\right\rangle, \quad \forall x, y \in A, a^{*}, b^{*} \in A^{*},
$$

is precisely equation (2.16).

The facts above and the results in the previous sections allow us to compare left-symmetric bialgebras and Lie bialgebras in terms of the following properties: structures on the corresponding Lie groups, 1-cocycles of Lie algebras, matched pairs of Lie algebras, Lie algebra structures on the direct sum of the Lie algebras in the matched pairs, bilinear forms on the direct sum of the Lie algebras in the matched pairs, double structures on the direct sum of the Lie algebras in the matched pairs, algebraic equations associated to coboundary cases, nondegenerate solutions and related geometric interpretation, $\mathcal{O}$-operators and constructions from left-symmetric algebras. We list them in Table 1. From this table, we observe that there is a clear analogy between them and in particular, parakähler Lie groups correspond to Poisson-Lie groups whose Lie algebras are Lie bialgebras in this sense.

Moreover, since classical Yang-Baxter equations can be regarded as "classical limits" of quantum Yang-Baxter equations ([Be]), we believe that there should exist an analogue ("quantum $S$-equations" ) of the quantum Yang-Baxter equations. We use a question mark in Table 1 to denote these still-to-be-found "quantum $S$-equations". 
Table 1: Comparison between Lie bialgebras and left-symmetric bialgebras

\begin{tabular}{|c|c|c|}
\hline Algebras & Lie bialgebras & Left-symmetric bialgebras \\
\hline Corresponding Lie groups & Poisson-Lie groups & parakähler Lie groups \\
\hline 1-cocycles of Lie algebras & $1 \otimes \mathrm{ad}+\mathrm{ad} \otimes 1$ & $L \otimes 1+1 \otimes$ ad \\
\hline Matched pairs of Lie algebras & $\left(\mathcal{G}, \mathcal{G}^{*}, \operatorname{ad}_{\mathcal{G}}^{*}, \operatorname{ad}_{\mathcal{G}^{*}}^{*}\right)$ & $\left(\mathcal{G}(A), \mathcal{G}\left(A^{*}\right), L_{A}^{*}, L_{A^{*}}^{*}\right)$ \\
\hline $\begin{array}{l}\text { Lie algebra structures on } \\
\text { the direct sum of the Lie } \\
\text { algebras in the matched pairs }\end{array}$ & Manin triples & phase spaces \\
\hline \multirow{3}{*}{$\begin{array}{l}\text { Bilinear forms on } \\
\text { the direct sum of the Lie } \\
\text { algebras in the matched pairs }\end{array}$} & symmetric & skew-symmetric \\
\hline & $\begin{aligned} &\left\langle x+a^{*}, y+b^{*}\right\rangle \\
&=\left\langle x, b^{*}\right\rangle+\left\langle a^{*}, y\right\rangle \\
&\end{aligned}$ & $\begin{aligned} &\left\langle x+a^{*}, y+b^{*}\right\rangle \\
&=-\left\langle x, b^{*}\right\rangle+\left\langle a^{*}, y\right\rangle \\
&\end{aligned}$ \\
\hline & invariant & 2-cocycles \\
\hline $\begin{array}{c}\text { Double structures on } \\
\text { the direct sum of the Lie } \\
\text { algebras in the matched pairs }\end{array}$ & Drinfeld Doubles & symplectic doubles \\
\hline \multirow{2}{*}{$\begin{array}{c}\text { Algebraic equations associated } \\
\text { to coboundary cases }\end{array}$} & skew-symmetric solutions & symmetric solutions \\
\hline & CYBEs in Lie algebras & $\begin{array}{c}S \text {-equations in left-symmetric } \\
\text { algebras }\end{array}$ \\
\hline \multirow[t]{2}{*}{ Nondegenerate solutions } & 2-cocycles of Lie algebras & $\begin{array}{c}\text { 2-cocycles of left-symmetric } \\
\text { algebras }\end{array}$ \\
\hline & symplectic structures & Hessian structures \\
\hline \multirow[t]{2}{*}{$\mathcal{O}$-operators } & associated to $\mathrm{ad}^{*}$ & associated to $L^{*}$ \\
\hline & skew-symmetric parts & symmetric parts \\
\hline \multirow{2}{*}{$\begin{array}{l}\text { Constructions from } \\
\text { left-symmetric algebras }\end{array}$} & $r=\sum_{i=1}^{n}\left(e_{i} \otimes e_{i}^{*}-e_{i}^{*} \otimes e_{i}\right)$ & $r=\sum_{i=1}^{n}\left(e_{i} \otimes e_{i}^{*}+e_{i}^{*} \otimes e_{i}\right)$ \\
\hline & $\begin{array}{c}\text { induced bilinear forms } \\
\quad\left\langle x+a^{*}, y+b^{*}\right\rangle \\
=-\left\langle x, b^{*}\right\rangle+\left\langle a^{*}, y\right\rangle \\
\end{array}$ & $\begin{array}{c}\text { induced bilinear forms } \\
\quad\left\langle x+a^{*}, y+b^{*}\right\rangle \\
=\left\langle x, b^{*}\right\rangle+\left\langle a^{*}, y\right\rangle \\
\end{array}$ \\
\hline Quantum equations & $\begin{array}{c}\text { Quantum Yang-Baxter } \\
\text { equations } \\
\end{array}$ & $?$ \\
\hline
\end{tabular}

At the end of this section, we consider the case that a left-symmetric bialgebra is also a Lie bialgebra.

Theorem 7.7 Let $\left(A, A^{*}, \alpha, \beta\right)$ be a left-symmetric bialgebra. Then $\left(\mathcal{G}(A), \mathcal{G}\left(A^{*}\right)\right)$ is a Lie bialgebra if and only if

$$
\left\langle R_{.}^{*}(x) a^{*}, R_{\circ}^{*}\left(b^{*}\right) y\right\rangle+\left\langle R_{.}^{*}(x) b^{*}, R_{\circ}^{*}\left(a^{*}\right) y\right\rangle=\left\langle R_{.}^{*}(y) b^{*}, R_{\circ}^{*}\left(a^{*}\right) x\right\rangle+\left\langle R_{.}^{*}(y) a^{*}, R_{\circ}^{*}\left(b^{*}\right) x\right\rangle,
$$

for any $x, y \in A^{*}, a^{*}, b^{*} \in A^{*}$.

Proof Denote the left-symmetric product on $A$ by "." and the left-symmetric product on $A^{*}$ by "o". Then $\left(\mathcal{G}(A), \mathcal{G}\left(A^{*}\right)\right)$ is a Lie bialgebra if and only if for any $x, y \in A, a^{*}, b^{*} \in A^{*}$,

$(*) \quad \operatorname{ad}_{.}^{*}(x)\left[a^{*}, b^{*}\right]-\left[\operatorname{ad}_{.}^{*}(x) a^{*}, b^{*}\right]-\left[a^{*}, \operatorname{ad}_{.}^{*}(x) b^{*}\right]+\operatorname{ad}_{.}^{*}\left(\operatorname{ad}_{\circ}^{*}\left(a^{*}\right) x\right) b^{*}-\operatorname{ad}_{.}^{*}\left(\operatorname{ad}_{\circ}^{*}\left(b^{*}\right) x\right) a^{*}=0$ 
$(* *) \operatorname{ad}_{\circ}^{*}\left(a^{*}\right)[x, y]-\left[\operatorname{ad}_{\circ}^{*}\left(a^{*}\right) x, y\right]-\left[x, \operatorname{ad}_{\circ}^{*}\left(a^{*}\right) y\right]+\operatorname{ad}_{\circ}^{*}\left(\operatorname{ad}_{.}^{*}(x) a^{*}\right) y-\operatorname{ad}_{\circ}^{*}\left(\operatorname{ad}_{.}^{*}(y) a^{*}\right) x=0$.

Since ad. $=L^{*}-R_{\text {. }}^{*}$ and $\left(\mathcal{G}(A), \mathcal{G}\left(A^{*}\right), L_{.}^{*}, L_{\circ}^{*}\right)$ is a matched pair of Lie algebras, the equation $(*)$ is reduced to

$$
\begin{aligned}
& -R_{\cdot}^{*}(x)\left[a^{*}, b^{*}\right]+\left[R_{\cdot}^{*}(x) a^{*}, b^{*}\right]+\left[a^{*}, R_{.}^{*}(x) b^{*}\right]-R_{.}^{*}\left(\operatorname{ad}_{\circ}^{*}\left(a^{*}\right) x\right) b^{*}-L_{.}^{*}\left(R_{\circ}^{*}\left(a^{*}\right) x\right) b^{*} \\
& +R_{.}^{*}\left(\operatorname{ad}_{\circ}^{*}\left(b^{*}\right) x\right) a^{*}+L_{.}^{*}\left(R_{\circ}^{*}\left(b^{*}\right) x\right) a^{*}=0 .
\end{aligned}
$$

Let the equation above act on $y \in A$ and note that $\left\langle R^{*}(x) a^{*}, y\right\rangle=\left\langle L^{*}(y) a^{*}, x\right\rangle$ for any $x, y \in A$ and $a^{*} \in A^{*}$, we obtain

$$
\begin{aligned}
& -L_{.}^{*}(y)\left[a^{*}, b^{*}\right]+L_{.}^{*}\left(\operatorname{ad}^{*}\left(b^{*}\right) y\right) a^{*}-L_{.}^{*}\left(\operatorname{ad}^{*}\left(a^{*}\right) y\right) b^{*}+\left[a, L_{.}^{*}(y) b^{*}\right]+R_{.}^{*}(y) b^{*} \circ a^{*} \\
& -\left[b^{*}, L_{.}^{*}(y) a^{*}\right]-R_{.}^{*}(y) a^{*} \circ b^{*}=0 .
\end{aligned}
$$

Using the condition that $\left(\mathcal{G}(A), \mathcal{G}\left(A^{*}\right), L_{.}^{*}, L_{\circ}^{*}\right)$ is a matched pair of Lie algebras again, we know that

$$
-L_{.}^{*}\left(R_{\circ}^{*}\left(b^{*}\right) y\right) a^{*}+L_{.}^{*}\left(R_{\circ}^{*}\left(a^{*}\right) y\right) b^{*}+R_{.}^{*}(y) b^{*} \circ a^{*}-R_{.}^{*}(y) a^{*} \circ b^{*}=0,
$$

which gives equation (7.9) by acting on $x \in A$. Similarly, from equation $(* *)$, we can get

$$
-L_{\circ}^{*}\left(R_{.}^{*}(y) b^{*}\right) x+L_{\circ}^{*}\left(R_{.}^{*}(x) b^{*}\right) y+R_{\circ}^{*}\left(b^{*}\right) y \cdot x-R_{\circ}^{*}\left(b^{*}\right) x \cdot y=0,
$$

for any $x, y \in A$ and $b^{*} \in A^{*}$, which gives the same equation (7.9) by acting on $a^{*} \in A^{*}$.

Corollary 7.8 Let $(A, \cdot)$ be a left-symmetric and $r \in A \otimes A$ be a symmetric solution of $S$-equation in $A$. Suppose the left-symmetric algebra structure on $A^{*}$ is induced by $r$ from equation (6.4). Then there exists a Lie bialgebra structure $\left(\mathcal{G}(A), \mathcal{G}\left(A^{*}\right)\right)$ if and only if

$$
\begin{aligned}
& -r\left(L_{.}^{*}(x) R_{\cdot}^{*}(y) a^{*}\right)+\left[x, r\left(R_{\cdot}^{*}(y) a^{*}\right]-\left(y \cdot r\left(a^{*}\right)-r\left(\operatorname{ad}_{\cdot}^{*}(y) a^{*}\right)\right) \cdot x\right. \\
= & -r\left(L_{.}^{*}(y) R_{.}^{*}(x) a^{*}\right)+\left[y, r\left(R_{.}^{*}(x) a^{*}\right]-\left(x \cdot r\left(a^{*}\right)-r\left(\operatorname{ad}_{.}^{*}(x) a^{*}\right)\right) \cdot y,\right.
\end{aligned}
$$

for any $x, y \in A$ and $a^{*} \in A^{*}$.

Corollary 7.9 Let $\left(A, A^{*}, \alpha, \beta\right)$ be a left-symmetric bialgebra. If equation (7.9) is satisfied, then there are two Lie algebra structures $\mathcal{G}(A) \bowtie_{L_{\circ}^{*}}^{L_{*}^{*}} \mathcal{G}\left(A^{*}\right)$ and $\mathcal{G}(A) \bowtie_{\mathrm{ad}_{\circ}^{*}}^{\text {ad }} \mathcal{G}\left(A^{*}\right)$ on the direct sum $A \oplus A^{*}$ of the underlying vector spaces of $A$ and $A^{*}$ such that both $\mathcal{G}(A)$ and $\mathcal{G}\left(A^{*}\right)$ are Lie subalgebras and the bilinear form given by equation $(2.16)$ is a 2-cocycle of $\mathcal{G}(A) \bowtie_{L_{\circ}^{*}}^{L^{*}} \mathcal{G}\left(A^{*}\right)$ and the bilinear form $(7.3)$ is invariant on $\mathcal{G}(A) \bowtie_{\mathrm{ad}_{\circ}^{*}}^{\mathrm{ad}_{*}^{*}} \mathcal{G}\left(A^{*}\right)$. 
Remark Obviously the two Lie algebras above are not isomorphic in general. On the other hand, if a Lie bialgebra $\left(\mathcal{G}, \mathcal{G}^{*}\right)$ whose Lie algebra structure on $\mathcal{G}^{*}$ is induced by a non-degenerate classical $r$-matrix $([\mathrm{D}],[\mathrm{Se}])$, then both $\mathcal{G}$ and $\mathcal{G}^{*}$ are symplectic Lie algebras. Therefore there is a compatible left-symmetric algebra structure on $\mathcal{G}$ and $\mathcal{G}^{*}$ respectively.

Example 7.1 Let $(\mathcal{G}, \omega)$ be a symplectic Lie algebra. Then there is a Lie bialgebra whose Lie algebra structure in $\mathcal{G}^{*}$ is given by a non-degenerate classical $r$-matrix as follows (cf. [DiM]).

$$
\delta(x)=[x \otimes 1+1 \otimes x, r], \quad \forall x \in \mathcal{G},
$$

where $r: \mathcal{G}^{*} \rightarrow \mathcal{G}$ is given by $\omega(x, y)=\left\langle r^{-1}(x), y\right\rangle$. On the other hand, there exists a leftsymmetric algebra structure "." on $\mathcal{G}$ given by equation $(2.14)$, that is, $\omega(x \cdot y, z)=-\omega(y,[x, z])$ for any $x, y, z \in \mathcal{G}$. Moreover, there exists a compatible left-symmetric algebra structure on the Lie algebra $\mathcal{G}^{*}$ given by

$$
a^{*} \circ b^{*}=r^{-1}\left(r\left(a^{*}\right) \cdot r\left(b^{*}\right)\right), \forall a^{*}, b^{*} \in \mathcal{G}^{*} .
$$

Furthermore, it is easy to know that

$\left.L_{.}^{*}(x) a^{*}=r^{-1}\left[x, r\left(a^{*}\right)\right], R_{.}^{*}(x) a^{*}=-r^{-1}\left(r\left(a^{*}\right) \cdot x\right)\right), L_{\circ}^{*}\left(a^{*}\right) x=\left[r\left(a^{*}\right), x\right], R_{\circ}^{*}\left(a^{*}\right) x=-x \cdot r\left(a^{*}\right)$,

for any $x \in A, a^{*} \in A^{*}$. Therefore according to Theorem $2.5,\left(\mathcal{G}, \mathcal{G}^{*}\right)$ (as left-symmetric algebras) is a left-symmetric bialgebra if and only if $\mathcal{G}$ is 2 -step nilpotent, that is, $[[x, y], z]=0$ for any $x, y, z \in \mathcal{G}$. In this case, we can know that it is equivalent to $[x, y] \cdot z=0$ for any $x, y, z \in \mathcal{G}$. Therefore, equation (7.9) holds naturally.

\section{Acknowledgments}

The author thanks Professors P. Etingof, I.M. Gel'fand, B.A. Kupershmidt, and C. Woodward for important suggestion and great encouragement. He is very grateful of referees' important suggestion. He also thanks Professors J. Lepowsky, Y.-Z. Huang and H.S. Li for the hospitality extended to him during his stay at Rutgers, The State University of New Jersey and for valuable discussions. This work was supported in part by S.S. Chern Foundation for Mathematical Research, the National Natural Science Foundation of China (10571091, 10621101), NKBRPC (2006CB805905), Program for New Century Excellent Talents in University and K.C. Wong Education Foundation. 


\section{References}

[AD] A. Andrada, I.G. Dotti, Double products and hypersymplectic structures on $\mathbf{R}^{4 n}$, arXiv: math.DG/0401294.

[AS] A. Andrada, S. Salamon, Complex product structure on Lie algebras, Forum Math. 17 (2005) 261-295.

[Bai1] C.M. Bai, Left-symmetric algebras from linear functions, J. Algebra 281 (2004)651-665.

[Bai2] C.M. Bai, A further study on non-abelian phase spaces: Left-symmetric algebraic approach and related geometry, Rev. Math. Phys. 18 (2006), 545-564.

[Bai3] C.M. Bai, A unified algebraic approach to the classical Yang-Baxter equation, to appear in J. Phys. A: Math. Theo. (2007).

[Bai4] C.M. Bai, Parakähler structures on Lie groups, preprint.

[BKL] C.M. Bai, L. Kong, H.S. Li, Novikov algebras and vertex algebras, preprint.

[BM1] C.M. Bai, D.J. Meng, The classification of Novikov algebras in low dimensions, J. Phys. A: Math. Gen. 34 (2001) 1581-1594.

[BM2] C.M. Bai, D.J. Meng, On the realization of transitive Novikov algebras, J. Phys. A: Math. Gen. 34 (2001) 3363-3372.

[BM3] C.M. Bai, D.J. Meng, The realizations of non-transitive Novikov algebras, J. Phys. A: Math. Gen. 34 (2001) 6435-6442.

[BM4] C.M. Bai, D.J. Meng, Bilinear forms on Novikov algebras, Int. J. Theor. Phys. 41 (2002) 495-502.

[BM5] C.M. Bai, D.J. Meng, A Lie algebraic approach to Novikov algebras, J. Geom. Phys. 45 (2003) 218-230.

[BK] B. Bakalov, V. Kac, Field algebras, Int. Math. Res. Not. (2003) 123-159.

[BN] A.A. Balinskii, S.P. Novikov, Poisson brackets of hydrodynamic type, Frobenius algebras and Lie algebras, Soviet Math. Dokl. 32 (1985) 228-231.

[Bar] M.L. Barbeis, Hypercomplex structures on four-dimensional Lie groups, Proc. Amer. Math. Soc. 125 (1997) 1043-1054.

[Bax] G. Baxter, An analytic problem whose solution follows from a simple algebraic identity, Pacific J. Math. 10 (1960) 731-742.

[Be] A.A. Belavin, Dynamical symmetry of integrable quantum systems, Nucl. Phys. B 180 (1981) 189-200. 
[BD] A.A. Belavin, V.G. Drinfeld, Solutions of classical Yang-Baxter equation for simple Lie algebras, Funct. Anal. Appl. 16 (1982) 159-180.

[Bo1] M. Bordemann, Generalized Lax pairs, the modified classical Yang-Baxter equation, and affine geometry of Lie groups, Comm. Math. Phys. 135 (1990) 201-216.

[Bo2] M. Bordemann, Nondegenerate invariant bilinear forms on nonassociative algebras, Acta Math. Univ. Comen. LXVI (1997) 151-201.

[Bu1] D. Burde, Simple left-symmetric algebras with solvable Lie algebra, Manuscripta Math. 95 (1998) 397-411.

[Bu2] D. Burde, Left-symmetric algebras, or pre-Lie algebras in geometry and physics, Cent. Eur. J. Math. 4 (2006) 323-357.

[Ca] A. Cayley, On the theory of analytic forms called trees, Collected Mathematical Papers of Arthur Cayley, Cambridge Univ. Press, Vol. 3 (1890) 242-246.

[CL] F. Chapoton, M. Livernet, Pre-Lie algebras and the rooted trees operad, Int. Math. Res. Not. (2001) 395-408.

[CP] V. Chari, A. Pressley, A guide to quantum groups, Cambridge University Press, Cambridge (1994).

[Ch] B.Y. Chu, Symplectic homogeneous spaces, Trans. Amer. Math. Soc. 197 (1974) 145-159.

[CK] A. Connes, D. Kreimer, Hopf algebras, renormalization and noncommutative geometry, Comm. Math. Phys. 199 (1998) 203-242.

[DaM1] J.M. Dardie, A. Medina, Double extension symplectique d'un groupe de Lie symplectique, Adv. Math. 117 (1996) 208-227.

[DaM2] J.M. Dardie, A. Medina, Algèbres de Lie kählériennes et double extension, J. Algebra 185 (1996) 774-795.

[DiM] A. Diatta, A. Medina, Classical Yang-Baxter equation and left-invariant affine geometry on Lie groups, arXiv:math.DG/0203198.

[D] V. Drinfeld, Hamiltonian structure on the Lie groups, Lie bialgebras and the geometric sense of the classical Yang-Baxter equations, Soviet Math. Dokl. 27 (1983) 68-71.

[DL] A. Dzhumadil'daev, C. Lofwall, Trees, free right-symmetric algebras, free Novikov algebras and identities, Homology, Homotopy and applications 4 (2002) 165-190.

[E] K. Ebrahimi-Fard, Loday-type algebras and the Rota-Baxter relation, Lett. Math. Phys. 61 (2002) 139-147. 
[EGK] K. Ebrahimi-Fard, L. Guo, D. Kreimer, Integrable renormalization 1: The ladder case, J. Math. Phys, 45 (2004) 3758-3769.

[ES] P. Etingof, A. Soloviev, Quantization of geometric classical r-matrix, Math. Res. Lett. 6 (1999) 223-228.

[FHL] I. Frenkel, Y.Z. Huang, J. Lepowsky, On axiomatic approaches to vertex operator algebras and modules, Memoris. Amer. Math. Soc. 104 (1993).

[FLM] I. Frenkel, J. Lepowsky, A. Meurman, Vertex operator algebras and the Monster, Pure and Appl. Math. 134, Academic Press, Boston (1988).

[F] V.T. Filipov, A class of simple nonassociative algebras, Mat. Zametki 45 (1989) 101-105.

[GD] I.M. Gel'fand, I. Ya. Dorfman, Hamiltonian operators and algebraic structures related to them, Funct. Anal. Appl. 13 (1979) 248-262.

[G] M. Gerstenhaber, The cohomology structure of an associative ring, Ann. Math. 78 (1963) 267-288.

[GS] I.Z. Golubschik, V.V. Sokolov, Generalized operator Yang-Baxter equations, integrable ODEs and nonassociative algebras, J. Nonlinear Math. Phys., 7 (2000) 184-197.

[H] J.I. Hano, On kaehlerian homogeneous spaces of unimodular Lie groups, Amer. J. Math. 79 (1957) 885-900.

[JL] J. Lepowsky, H.S. Li, Introduction to vertex operator algebras and their representations, Progress in Mathematics 227, Birkäuser, Boston (2004).

[Ka] S. Kaneyuki, Homogeneous symplectic manifolds and dipolarizations in Lie algebras, Tokyo J. Math. 15 (1992) 313-325.

[KGM] Y. Khakimdjanov, M. Goze and A. Medina, Symplectic or contact structures on Lie groups, arXiv:math.DG/0205290.

[Ki] H. Kim, Complete left-invariant affine structures on nilpotent Lie groups, J. Diff. Geom. 24 (1986) 373-394.

[KM] V. Kosmann-Schwarzbach, F. Magri, Poisson-Lie groups and complete integrability, I. Drinfeld algebras, dual extensions and their canonical representations, Ann. Inst. Henri Poincaré Phys. Théor 49 (1988) 35-81.

[Ko] J.-L. Koszul, Domaines bornés homogènes et orbites de groupes de transformations affines, Bull. Soc. Math. France 89 (1961) 515-533.

[Ku1] B.A. Kupershmidt, Non-abelian phase spaces, J. Phys. A: Math. Gen. 27 (1994) 28012810. 
[Ku2] B.A. Kupershmidt, On the nature of the Virasoro algebra, J. Nonlinear Math. Phy. 6 (1999) 222-245.

[Ku3] B.A. Kupershmidt, What a classical $r$-matrix really is, J. Nonlinear Math. Phy. 6 (1999) 448-488.

[Li] H.S. Li, Vertex algebras and vertex Poisson algebras, Comm. Contemp. Math. 6 (2004) $61-110$.

[Lib] P. Libermann, Sur le problème d'équivalence de certaines structures infinitésimals, Ann. Mat. Pura Appl. 36 (1954) 27-120.

[LM] A. Lichnerowicz, A. Medina, On Lie groups with left invariant symplectic or kahlerian structures, Lett. Math. Phys. 16 (1988) 225-235.

[LW] J.H. Lu, A. Weinstein, Poisson-Lie groups, dressing transformations and Bruhat decompositions, J. Diff. Geom 31 (1990) 501-526.

[Maj] S. Majid, Matched pairs of Lie groups associated to solutions of the Yang-Baxter equations, Pacific J. Math. 141 (1990) 311-332.

[Mat] Y. Matsushima, Affine structures on complex mainfolds, Osaka J. Math. 5 (1968) 215-222.

[MS] D. Mcduff, D. Salamon, Introduction to symplectic topology, Clarendon Press, Oxford, (1998).

[Me] A. Medina, Flat left-invariant connections adapted to the automorphism structure of a Lie group, J. Diff. Geom. 16 (1981) 445-474.

[R] G.-C. Rota, Baxter operators, an introduction, In: "Gian-Carlo Rota on Combinatorics, Introductory papers and commentaries", Joseph P.S. Kung, Editor, Birkhäuser, Boston, 1995.

[SW] A.A. Sagle, R.E. Walde, Introduction to Lie groups and Lie algebras, Academic Press, New York (1973).

[Sc] R. Schafer, An introduction to nonassociative algebras, Dover Publications Inc., New York (1995).

[Se] M.A. Semonov-Tian-Shansky, What is a classical R-matrix? Funct. Anal. Appl. 17 (1983) $259-272$.

[Sh] H. Shima, Homogeneous Hessian manifolds, Ann. Inst. Fourier 30 (1980) 91-128.

[SS] S.I. Svinolupov, V.V. Sokolov, Vector-matrix generalizations of classical integrable equations, Theoret. and Math. Phys. 100 (1994) 959-962. 
[T] M. Takeuchi, Matched pairs of groups and bimash products of Hopf algebras, Comm. Algebra 9 (1981) 841-842.

[V] E.B. Vinberg, Convex homogeneous cones, Transl. of Moscow Math. Soc. No. 12 (1963) 340-403.

[W] A. Winterhalder, Linear Nijenhuis-tensors and the construction of integrable systems, arXiv: physics/9709008

[X] X. Xu, On simple Novikov algebras and their irreducible modules, J. Algebra 185 (1996) 905-934. 